\title{
Integrated sRNAome and RNA-Seq analysis reveals miRNA effects on betalain biosynthesis in pitaya
}

\author{
Canbin Chen ${ }^{1}$, Fangfang Xie ${ }^{1}$, Qingzhu Hua ${ }^{1}$, Noemi Tel-Zur², Lulu Zhang ${ }^{1}$, Zhike Zhang ${ }^{1}$, Rong Zhang ${ }^{1}$, \\ Jietang Zhao ${ }^{1}$, Guibing $\mathrm{Hu}^{1}$ and Yonghua Qin ${ }^{1 *}$ (D)
}

\begin{abstract}
Background: MicroRNAs (miRNAs) and their regulatory functions in anthocyanin, carotenoid, and chlorophyll accumulation have been extensively characterized in many plant species. However, the miRNA regulatory mechanism in betalain biosynthesis remains mostly unknown.

Results: In this study, 126 conserved miRNAs and 41 novel miRNAs were first isolated from Hylocereus monacanthus, among which 95 conserved miRNAs belonged to 53 miRNA families. Thirty-four candidate miRNAs related to betalain biosynthesis were differentially expressed. The expression patterns of those differential expressed miRNAs were analyzed in various pitaya tissues by RT-qPCR. A significantly negative correlation was detected between the expression levels of half those miRNAs and corresponding target genes. Target genes of miRNAs i.e. Hmo-miR157b-HmSPL6-like, Hmo-miR160a-Hpcyt P450-like3, Hmo-miR6020-HmCYP71A8-like, Hmo-novel-2HmCYP83B1-like, Hmo-novel-15-HmTPST-like, Hmo-miR828a-HmTT2-like, Hmo-miR858-HmMYB12-like, Hmo-miR858HMMYBC1-like and Hmo-miR858-HmMYB2-like were verified by 5'RACE and transient expression system in tobacco.

Conclusions: Hmo-miR157b, Hmo-miR160a, Hmo-miR6020 Hmo-novel-2, Hmo-novel-15, Hmo-miR828a and HmomiR858 play important roles in pitaya fruit coloration and betalain accumulation. Our findings provide new insights into the roles of miRNAs and their target genes of regulatory functions involved in betalain biosynthesis of pitaya.
\end{abstract}

Keywords: Hylocereus, Betalain biosynthesis, sRNAome and RNA-Seq, miRNA, Gene expression, 5'RACE

\section{Background}

Mature microRNAs (miRNAs) are a type of endogenous non-coding small RNAs with 20-24 nucleotide (nt) length. miRNAs regulate their target genes by mRNA at the posttranscriptional level via the RNA-induced silencing complex (RISC) by binding with the Argonaute (AGO) protein

\footnotetext{
* Correspondence: qinyh@scau.edu.cn

${ }^{1}$ State Key Laboratory for Conservation and Utilization of Subtropical Agrobioresources/Guangdong Provincial Key Laboratory of Postharvest Science of Fruits and Vegetables/Key Laboratory of Biology and Genetic Improvement of Horticultural Crops (South China), Ministry of Agriculture and Rural Affairs, College of Horticulture, South China Agricultural University, Guangzhou, Guangdong 510642, P. R. China

Full list of author information is available at the end of the article
}

to cleavage target mRNA or repress translation of target mRNA [1]. miRNAs play vital roles in plant growth and development, (a)biotic stress response, post-transcriptional regulation, and pigment regulation [2-4].

miRNAs are involved in chlorophyll, carotenoid, and anthocyanin biosynthesis. miRNAs can regulate coloration and chlorophyll accumulation [3, 5-7]. Overexpression of osa-miR171b by an artificial miRNA could enhance chlorophyll accumulation in rice leaves [7]. Based on the reduction of chlorophyll concentration under severe drought stress, a miRNA regulatory network consisting of two upregulated miRNAs and nineteen down-regulated miRNAs was constructed in Camellia sinensis [3]. miRNAs are

(c) The Author(s). 2020, corrected publication 2023. Open Access This article is licensed under a Creative Commons Attribution 4.0 International License, which permits use, sharing, adaptation, distribution and reproduction in any medium or format, as long as you give appropriate credit to the original author(s) and the source, provide a link to the Creative Commons licence, and indicate if changes were made. The images or other third party material in this article are included in the article's Creative Commons licence, unless indicated otherwise in a credit line to the material. If material is not included in the article's Creative Commons licence and your intended use is not permitted by statutory regulation or exceeds the permitted use, you will need to obtain permission directly from the copyright holder. To view a copy of this licence, visit http://creativecommons.org/licenses/by/4.0/. The Creative Commons Public Domain Dedication waiver (http://creativecommons.org/publicdomain/zero/1.0/) applies to the data made available in this article, unless otherwise stated in a credit line to the data. 
involved in regulating carotenoid pathways [8-12]. miR1857 affected carotenogenesis in a sweet orange redflesh mutant and its wild type [8]. In different rose cultivars, miRNAs may negatively regulate target genes to prevent carotenoid accumulation resulting in white flowers according to expression analyses of five miRNAs [9]. miRNAs can also regulate anthocyanin accumulation through their target genes [13-25]. Anthocyanin accumulation is promoted with increasing of miR156 abundance in Arabidopsis [13] and litchi [20]. However, the reduction of miR156 abundance could lead to the accumulation of flavonols [13]. miR156 positively regulates anthocyanin accumulation by targeting the SPL transcription factors (TFs) [23]. miR828 negatively regulates anthocyanin accumulation by inhibiting the expression of MYB75, MYB90, and MYB113 in Arabidopsis [14]. Moreover, miR858 and its targets involved in anthocyanin accumulation have been identified from apple, cotton, and tomato $[15,17,18]$.

Pitaya, also known as pitahaya or dragon fruit, is a perennial climbing fruit crop belonging to the genus Hylocereus (Cactaceae) under the order Caryophyllales. As a member of the Cactaceae, pitaya exhibits a range of specific adaptations to arid lands in term of succulent stems with spines instead of leaves, the crassulacean acid metabolism (CAM) pathway [26-28]. It is an excellent plant material for basic and applied biological research. The potential economic impact of pitaya lies in its diverse uses not only as agricultural produce and processed foods but also in industrial and medicinal products. Pitaya is a fast-return fruit crop with production in the second year after planting and full production in $3-4$ years. Pitaya fruit is mature in $28-50$ days (28-35 days in summer and 35-50 days in autumn) after flowering and has 7-12 separate fruiting cycles per year due to climatic or nutritional limitations in South China. Therefore, pitaya has become a favorite fruit of many farmers and home gardeners in Southeast Asia, China, the United States, Israel, Australia, Cyprus and the Canary Islands.

The color of pitaya is attributed to the presence of betalains [29-32]. Betalains are red and yellow alkaloid pigments that are found in all families of the Caryophyllales with the exception of Molluginaceae and Caryophyllaceae which produce anthocyanins [33]. Betalains in pitaya fruit are not only good for human health but also can help consumers distinguish cultivars $[29,34,35]$. Betalains also play vital roles in the protection against drought, UV radiation, high saline soils, and diseases [35-39]. Pitaya is the only at large-scale commercially grown fruit containing abundant betalains for the consumer. Previous studies are mainly focused on characterizations of key genes and TFs involved in betalain biosynthesis. Key genes such as tyrosinase (TYR), cytochrome P450 (Cyt P450), 4,5-dihydroxy-phenylalanine (DOPA)-dioxygenase $(D O D)$ and glucosyltransferases (GTs) [40] and TFs such as WRKY and MYB involved in betalain biosynthesis have been investigated in detail [41-43]. However, the roles of miRNAs in betalain biosynthesis has not been reported yet. In this study, candidate miRNAs and their target genes related to betalain biosynthesis were identified based on small RNA and transcriptome databases of pitaya pulp at different developmental stages. The aim of the present study is to explore the roles of miRNA in pitaya betalain biosynthesis, which may contribute to a better understanding of betalain biosynthesis in Hylocereus.

\section{Results}

\section{Sequencing of sRNAs and the transcriptome}

Six sRNA libraries were generated to identify miRNAs using pulps from 'Guanhuahong' pitaya on the $19^{\text {th }}$ day after flowering (DAF) (white pulp stage, Hp19d_1, and Hp19d_2), $25^{\text {th }}$ DAF (pulp coloration stages, Hp25d_1, and Hp25d_2) and $29^{\text {th }}$ DAF (mature stage, Hp29d_1, and Hp29d 2). The Illumina sequencing data of sRNAs from the $19^{\text {th }}, 25^{\text {th }}$ and $29^{\text {th }}$ DAF showed that 24 nt sRNAs are the most abundant, followed by $21 \mathrm{nt}$ sRNAs (Figure S1). A total of 82,318,241 reads were obtained from the sRNA datasets. After removal of the adaptor, insert, polyA and short RNAs of $<18 \mathrm{nt}$ in length, 62,729,725 (76.20\%) valid reads were obtained, including the rRNA, tRNA, snRNA, snoRNA and some other Rfam RNA (Table S1).

Three transcriptome libraries were constructed to identify the target genes of miRNAs using pulps from 'Guanhuahong' pitaya on the $19^{\text {th }}$ DAF (white pulp stage, Hp19d), $25^{\text {th }}$ DAF (pulp coloration stages, Hp25d) and $29^{\text {th }}$ DAF (mature stage, Hp29d). All screened reads were de novo assembled into 68,505 transcripts with an N50 of $1700 \mathrm{bp}$. And then, these transcripts were assembled into 39,737 unique sequences with an average length of $879 \mathrm{bp}$. The length distributions of those transcripts and unigenes were shown in Figure S2. The distribution of assembled transcripts and genes with different GC contents in the transcriptome datasets were summarized in Figure S3. The majority of transcripts and genes were in the range of 35$50 \%$ in GC contents.

\section{Identification of miRNAs}

A total of 126 conserved miRNAs were identified based on BLAST searches and sequence analyses in comparison of sRNA sequences with known mature plant miRNAs in miRBase (Table S2). Among them, 95 known miRNAs belonging to 53 families were obtained. The number of miRNA members for each family varied from 1 (for MIR535) to 7 (for MIR482) (Figure S4). Novel miRNAs were predicted using the sRNA valid reads based on structure and expression criteria [44]. Fortyone novel candidate miRNAs with a clear precursor including stem-loop secondary structure were identified (Table S2). The length of novel miRNAs was between 20 and $25 \mathrm{nt}$. The most abundant sequences (49\%) were $21 \mathrm{nt}$ - 
length, and followed by 24 nt-length (29\%), which was consistent with typical length distribution of mature miRNAs. The higher expression levels of the miRNA, the more copies of the miRNAs were sequenced. The abundance of known miRNAs was higher than that of putative novel miRNAs, except Hmo-novel-2, Hmo-novel-6, Hmo-novel-21 and Hmo-novel-22, which possessed more than 100 normalized reads (Table $\mathrm{S} 2$ ).

\section{Analyses of differentially expressed miRNAs}

High-throughput sequencing (HTS) was performed to explore the expression changes of miRNAs involved in betalain biosynthesis of 'Guanhuahong' pitaya. Only miRNAs with expression values at $p<0.05$ were considered to be significantly regulated. In $\mathrm{Hp} 29 \mathrm{~d} / \mathrm{Hp} 25 \mathrm{~d} /$ Hp19d, 33 known miRNAs and five novel miRNAs were found to be differentially expressed (Table 1).

Changes in expression levels of miRNAs during fruit development of pitaya possibly reflected their potential functional differences. In different pulp coloration stages of 'Guanhuahong' pitaya, the expression levels of Hmo-miR396b, HmomiR535, Hmo-novel-7 and Hmo-novel-12 at the red pulp stages $\left(25^{\text {th }}\right.$ and $29^{\text {th }}$ DAP) were significantly higher than that of the white pulp stage (19 $9^{\text {th }}$ DAP) (Table 1). Expression levels of Hmo-miR156, Hmo-miR157b, Hmo-miR164a, Hmo-miR164b and Hmo-miR399a increased gradually from $19^{\text {th }}$ DAF to $29^{\text {th }}$ DAF in 'Guanhuahong' pitaya. Those results were in consistent with betalain accumulation pattern $[45,46]$. However, the expression levels of Hmo-miR171c, Hmo-miR408, Hmo-miR393, Hmo-miR398c, HmomiR8175, Hmo-miR398a, Hmo-miR168a, Hmo-miR529b, Hmo-miR398b, Hmo-miR172a, Hmo-miR159a and HmomiR397b decreased gradually from $19^{\text {th }}$ DAF to $29^{\text {th }}$ DAF during fruit maturation of 'Guanhuahong' pitaya. Twelve differentially expressed miRNAs i.e. Hmo-miR390a, HmomiR6149a, Hmo-miR159b, Hmo-miR530, Hmo-miR6300, Hmo-miR390b, Hmo-miR6020, Hmo-miR394, HmomiR5072, Hmo-novel-15, Hmo-miR171d and Hmo-miR482f showed a lower expression level at the red pulp stages $\left(25^{\text {th }}\right.$ and $29^{\text {th }}$ DAP) compared to a relatively higher expression level at white pulp stage $\left(19^{\text {th }} \mathrm{DAP}\right)$. Those results were in contrast to betalain accumulation pattern suggesting that those miRNAs negatively regulated their target genes in betalain accumulation.

\section{Bioinformatics of transcriptome analyses}

All 39,737 unique sequences were annotated according to BLAST (cut-off E-value $\leq 10^{-5}$ ) searches of Nr, Swiss-Prot Protein, Pfam, Gene Ontology (GO), euKaryotic Ortholog Groups (KOG) and Kyoto Encyclopedia of Genes and Genomes (KEGG) database (Table S3).

The GO, KEGG and KOG databases were used to classify the functions of the predicted unigenes. Twelve thousand five hundred fifty-nine unigenes were classified into three main categories: 'biological process', 'cellular component', and 'molecular function' by GO database (Fig. 1). As for the 'molecular function' category, the largest number of unigenes was gathered in 'ATP binding', while the major groups for the 'cellular component' category were 'integral to membrane' (2878 unigenes, 23\%), 'nucleus' (2211 unigenes, 18\%) and 'plasma membrane' (1646 unigenes, 13\%). In the category of 'biological process', 'regulation of transcription, DNA-dependent' (729 unigenes, 5.8\%) and 'transcription, DNA-dependent' (706 unigenes, 5.6\%) were the two most abundant subcategories.

Seven thousand eight hundred twenty-two unigenes were mapped onto KEGG pathways. The highest number of unigenes was carbohydrate metabolism (796 unigenes), followed by energy metabolism (596 unigenes), amino acid metabolism (595 unigenes) and translation (510 unigenes) (Fig. 2). Differential expression genes (DEGs) in the Hp25dVS-Hp19d, Hp29d-VS-Hp19d, and Hp29d-VS-Hp25d were analyzed. In the Hp25d-VS-Hp19d, 24, 20, 16 and 13 DEGs were involved in phenylpropanoid, stilbenoid, diarylheptanoid and gingerol biosynthesis, DNA replication and flavonoid biosynthesis pathway $(p<0.0001)$ (Fig. 3a), respectively. In Hp29d-VS-Hp19d, 140 genes were found to be significantly differentially expressed $(\mathrm{p}<0.0001)$, including biosynthesis of phenylpropanoid, flavonoid, stilbenoid, diarylheptanoid and gingerol, metabolism of phenylalanine, $\alpha$-linolenic acid, starch and sucrose, xenobiotics by Cyt P450, as well as drug metabolism-Cyt P450 (Fig. 3b). The expression levels of DEGs among different groups in one pathway showed different scales of changes. Thirteen DEGs were detected from the flavonoid biosynthesis pathway in the Hp25d-VS-Hp19d and Hp29d-VS-Hp19d, respectively. However, no DEGs related to flavonoid biosynthesis were found in Hp29d-VS-Hp25d (Fig. 3c). Those results suggested that flavonoid biosynthesis is a crucial pathway in pitaya betalain biosynthesis.

The KOG database is used to study the classification and evolutionary rates of orthologous proteins. As shown in Fig. 4, group R (general function prediction only), group $\mathrm{O}$ (posttranslational modification, protein turnover, chaperones) and group $\mathrm{T}$ (signal transduction mechanisms) are the three most abundant groups in pitaya dataset, suggesting that a large number of transcriptional and posttranslational regulation of gene expression and function are involved in pitaya fruit development.

DEGs from three development stages (Hp25d-VSHp19d, Hp29d-VS-Hp19d, and Hp29d-VS-Hp25d) were evaluated by pairwise comparisons using the expression fold ( $\mid \log _{2}$ fold change $\mid \geq 1$ ) and $p$ values $<0.05$ as the thresholds (Fig. 5). In the pairwise comparisons between any two stages, 3988 genes were found to be significantly differentially expressed. The highest amount of DEGs was obtained between the Hp19d and Hp29d libraries, including 1486 down-regulated and 733 up-regulated 
Table 1 The information of differentially expressed miRNAs in pitaya

\begin{tabular}{|c|c|c|c|c|c|}
\hline miRNA names & miRNA sequences & Pvalue (ANOVA) & Hp19d (norm) & Hp25d (norm) & Hp29d (norm) \\
\hline Hmo-MIR2916-p5 & TACCGTCCTAGTCTCAACCATA & $2.99 \mathrm{E}-07$ & 807 & 141 & 2298 \\
\hline Hmo-miR171c & TTGAGCCGCGCCAATATCACC & 1.97E-05 & 156 & 72 & 39 \\
\hline Hmo-MIR2916-p3 & CAGGGATCGGCGGATGTTGCT & $1.38 \mathrm{E}-04$ & 2332 & 738 & 3765 \\
\hline Hmo-miR408 & TTGCACTGCCTCTTCCCTGGC & $5.14 \mathrm{E}-04$ & 682 & 103 & 68 \\
\hline Hmo-miR396b & CGGTTCAATAAAGCTGTGGGA & $8.78 \mathrm{E}-04$ & 193 & 334 & 332 \\
\hline Hmo-miR393 & TCCAAAGGGATCGCATTGATCC & $1.98 \mathrm{E}-03$ & 697 & 560 & 405 \\
\hline Hmo-miR164b & CATGTGCCTGTCTTCCCCATC & $2.20 \mathrm{E}-03$ & 1852 & 7112 & 24,055 \\
\hline Hmo-miR398c & TGTGTTCTCAGGTCGCCCCTG & $2.58 \mathrm{E}-03$ & 650 & 136 & 66 \\
\hline Hmo-miR390a & CGCTATCCATCCTGAGTTTCA & $2.75 \mathrm{E}-03$ & 187 & 57 & 85 \\
\hline Hmo-miR168a & GATCCCGCCTTGCATCAATTGAAT & $3.04 \mathrm{E}-03$ & 193 & 139 & 102 \\
\hline Hmo-miR529b & AGAAGAGGGAGAGTACAGCT & $3.81 \mathrm{E}-03$ & 1970 & 853 & 572 \\
\hline Hmo-miR398b & TGTGTTCTCAGGTCGCCCC & $4.13 \mathrm{E}-03$ & 89 & 11 & 7 \\
\hline Hmo-miR164a & TGGAGAAGCAGGGCACGTGCA & $4.28 \mathrm{E}-03$ & 4479 & 27,331 & 89,762 \\
\hline Hmo-novel-7 & TTACTTGGCACTTACGACAGA & $4.32 \mathrm{E}-03$ & 29 & 63 & 59 \\
\hline Hmo-miR399a & CGCCAAAGGAGAGTTGCCCTT & 4.40E-03 & 4 & 10 & 18 \\
\hline Hmo-miR8175 & GTTCGATCCCCGGCAACGGCGCCA & 4.40E-03 & 33 & 7 & 4 \\
\hline Hmo-miR156 & TTGCCAGAAGAGAGTGAGCAC & $6.04 \mathrm{E}-03$ & 30 & 65 & 75 \\
\hline Hmo-miR6149a & TTGATACGCACCTGAATCGGC & $6.08 \mathrm{E}-03$ & 38 & 7 & 10 \\
\hline Hmo-miR172a & CGAATCTTGATGATGCTGCAT & 7.33E-03 & 461 & 369 & 227 \\
\hline Hmo-miR397b & TTGAGTGCAGCGTTGATGAAAT & $7.48 \mathrm{E}-03$ & 31 & 5 & 1 \\
\hline Hmo-miR535 & TGACAACGAGAGAGAGCACGC & 8.93E-03 & 3907 & 10,306 & 9059 \\
\hline Hmo-miR159b & GGCTTGGATTGAAGGGAGCTCC & 1.17E-02 & 20 & 7 & 9 \\
\hline Hmo-miR159a & TTGGATTGAAGGGAGCTCTA & $1.20 \mathrm{E}-02$ & 198 & 182 & 108 \\
\hline Hmo-miR530 & TGCATTTGCACCTGCACCTGA & $1.71 \mathrm{E}-02$ & 15 & 5 & 8 \\
\hline Hmo-miR6300 & GTCGTTGTAGTATAGTGG & 1.80E-02 & 2638 & 88 & 161 \\
\hline Hmo-novel-21 & ACGCCTAATGCTGTGTATGGGAGG & $1.90 \mathrm{E}-02$ & 179 & 222 & 118 \\
\hline Hmo-miR390b & AAGCTCAGGAGGGATAGCGCC & $1.91 \mathrm{E}-02$ & 29 & 5 & 6 \\
\hline Hmo-miR398a & TGTGTTCTCAGGTCACCCCTT & 1.93E-02 & 64 & 27 & 25 \\
\hline Hmo-novel-2 & CAGCTTTCTTGAACTTTCCCC & 2.23E-02 & 566 & 501 & 773 \\
\hline Hmo-miR394 & TGGGCATTCTGTCCACCTCC & $2.38 \mathrm{E}-02$ & 6 & 2 & 2 \\
\hline Hmo-miR157b & CTGCCAGAAGATAGAGAGCAC & $2.38 \mathrm{E}-02$ & 50 & 105 & 148 \\
\hline Hmo-miR6020 & AAATGTTCTTCGAGTATCTTC & $2.51 \mathrm{E}-02$ & 5 & 0 & 1 \\
\hline Hmo-miR160b & TGCCTGGCTCCCTGTATGCCG & 2.89E-02 & 164 & 189 & 76 \\
\hline Hmo-miR5072 & TCCCCAGTGGAGTCGCCA & $2.95 \mathrm{E}-02$ & 21 & 2 & 3 \\
\hline Hmo-novel-15 & TCGCGCCTCGGGACCCTTTGC & 2.99E-02 & 48 & 31 & 32 \\
\hline Hmo-novel-12 & AGAGAAAGCATAAGCAACTGT & $3.28 \mathrm{E}-02$ & 0 & 8 & 6 \\
\hline Hmo-miR171d & TTGAGCCGTGCCAATATCCCA & 4.38E-02 & 9 & 2 & 3 \\
\hline Hmo-miR482f & GGTATTGGTGGGTTGGAAAGC & 4.88E-02 & 2 & 0 & 0 \\
\hline
\end{tabular}

(Fig. 5a). The lowest number of DEGs (1835) was detected between the Hp25d and Hp29d libraries (987 down-regulated and 848 up-regulated), followed by the Hp19d and Hp25d libraries (1961 DEGs including 1274 down-regulated and 687 up-regulated). Among those DEGs, 124 genes were significantly differentially expressed in all three fruit development stages of 'Guanhuahong' pitaya (Fig. 5b).
Verification of the accuracy of the sRNAome and RNA-Seq data

To verify the reliability of the sRNAome and RNA-Seq results, the expression of fifteen miRNAs and fifteen genes related to betalain biosynthesis were analyzed by RT-qPCR. The IDs, Reads Per Kilobase of exon model per Million mapped read (RPKM) value, and primers for the 30 transcripts were shown in Tables S2, S5, S6, and 


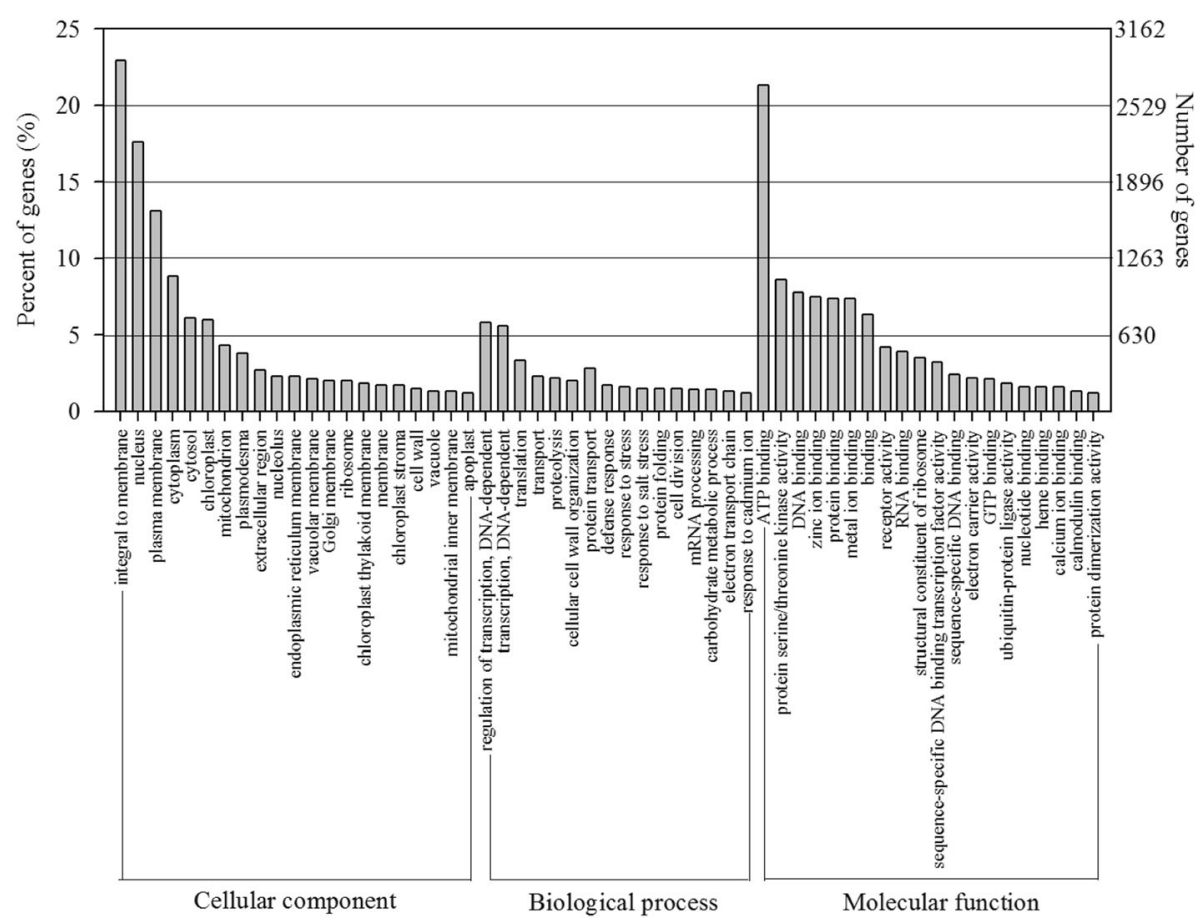

Fig. 1 Histogram of GO classifications for transcripts of pitaya pulp

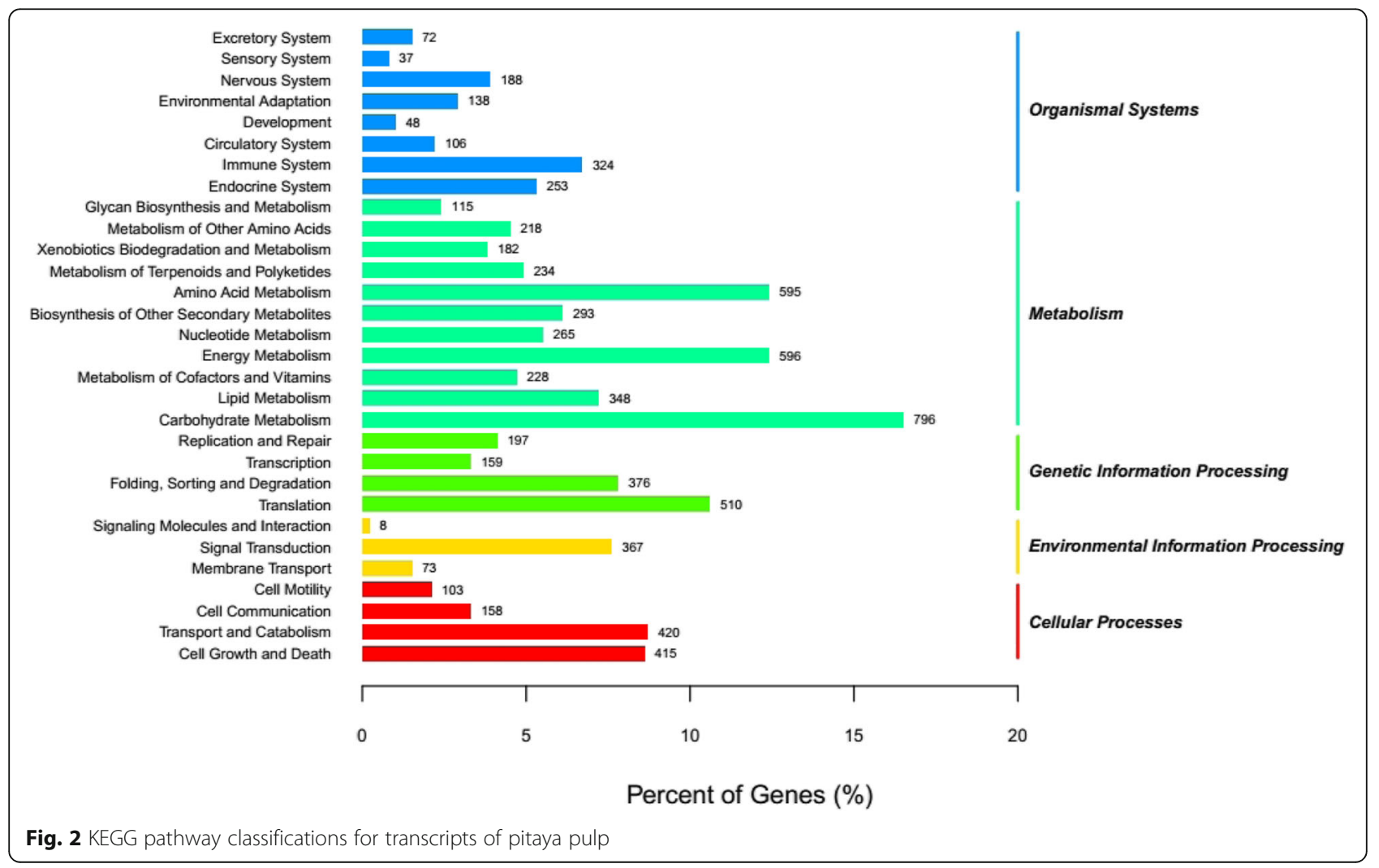




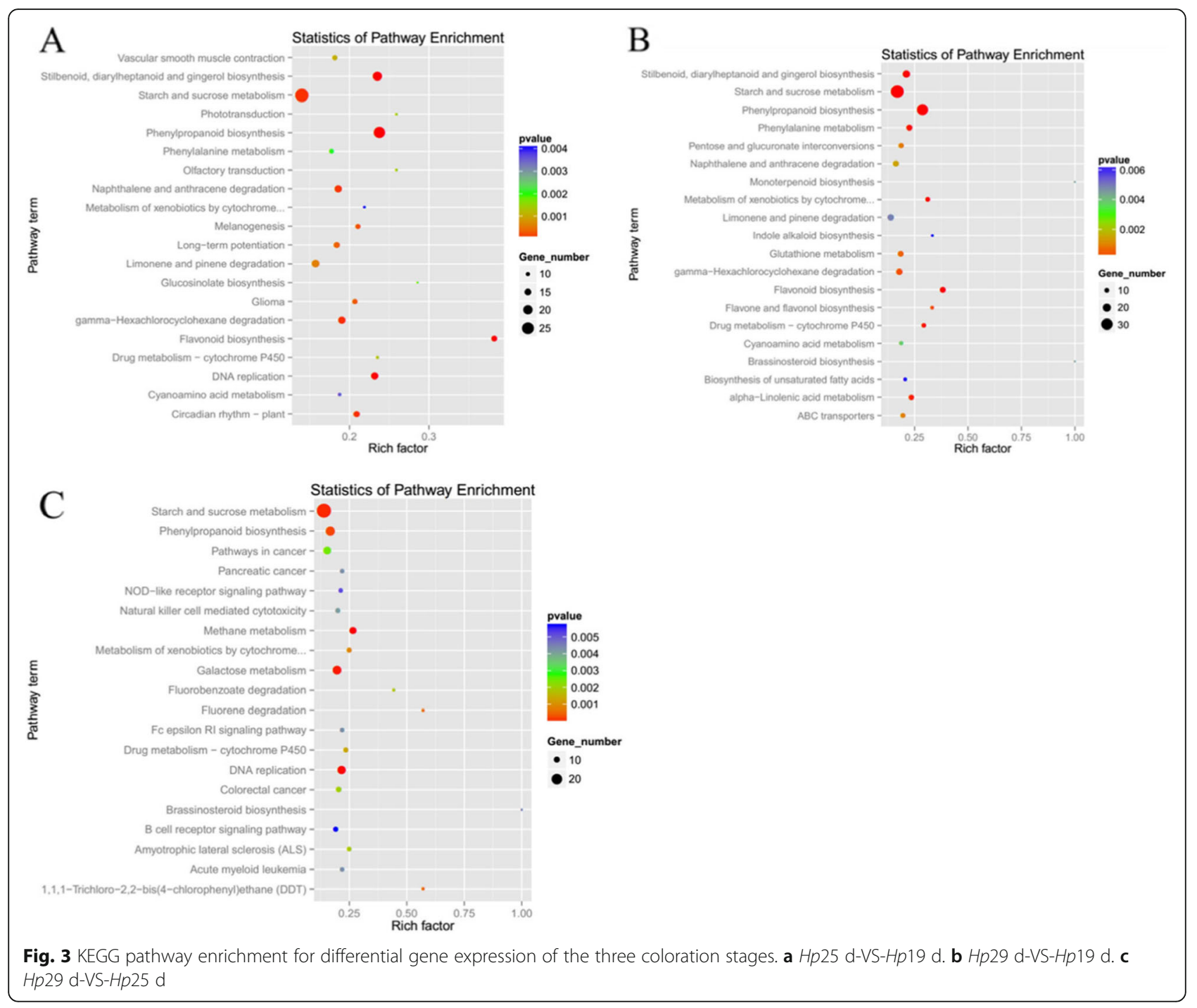

S8, respectively. The overall correlation coefficients of $0.717^{* * *}$ and $0.719^{* * *}$ were obtained by linear regression $[(\mathrm{Q}-\mathrm{PCR}$ value $)=\mathrm{a}($ sRNAome or RNA-Seq value $)+\mathrm{b}]$ analysis (Fig. 6), respectively, indicating that the results of sRNAome and transcriptome were consistent with those of RT-qPCR. Those results suggested that sRNAome and RNA-Seq data can be used for subsequent experiments.

\section{Prediction of targets for differentially expressed miRNAs}

To investigate the functions of miRNAs in betalain biosynthesis of pitaya, it is crucial to predict their target genes. Target genes of differentially-expressed miRNAs were predicted by Target Finder software. Transcripts of transcriptome were identified as possible target genes for the majority of miRNAs. A total of 124 target mRNAs, 22 target mRNAs for 25 conserved miRNAs and 5 novel miRNAs were obtained, respectively (Table S9). More than one target gene was predicted for most miRNAs. miRNAs have the potential to regulate targets belonging to certain gene families with different biological functions. For example, the predicted target genes of Hmo-miR529b were found to be involved in squamosa promoter-binding-like protein 6 (SPL6), histone-lysine N-methyltransferase and ubiquitinprotein ligase. Based on transcriptome annotation, six targets involved in betalain biosynthesis were selected [23, 40-42]. Among them, HmCYP83B1-like, HmCYP71A8-like, HmTPST-like, HmWDTC1-like and HmSPL16-like were respectively targeted by Hmo-novel-2, Hmo-miR6020, Hmo-novel-15, Hmo-miR164a and Hmo-miR156 while HmSPL6-like was co-targeted by Hmo-miR156, HmomiR157b and Hmo-miR529b.

Tissue-specific analyses of differentially expressed miRNAs miRNA preferentially expressed in specific tissues can provide clues to its physiological functions. Thirty differentially expressed miRNAs were analyzed their functions by RT-qPCR using ten different tissues from 'Guanhuabai' and 'Guanhuahong' pitayas. As shown in Fig. 7, the 


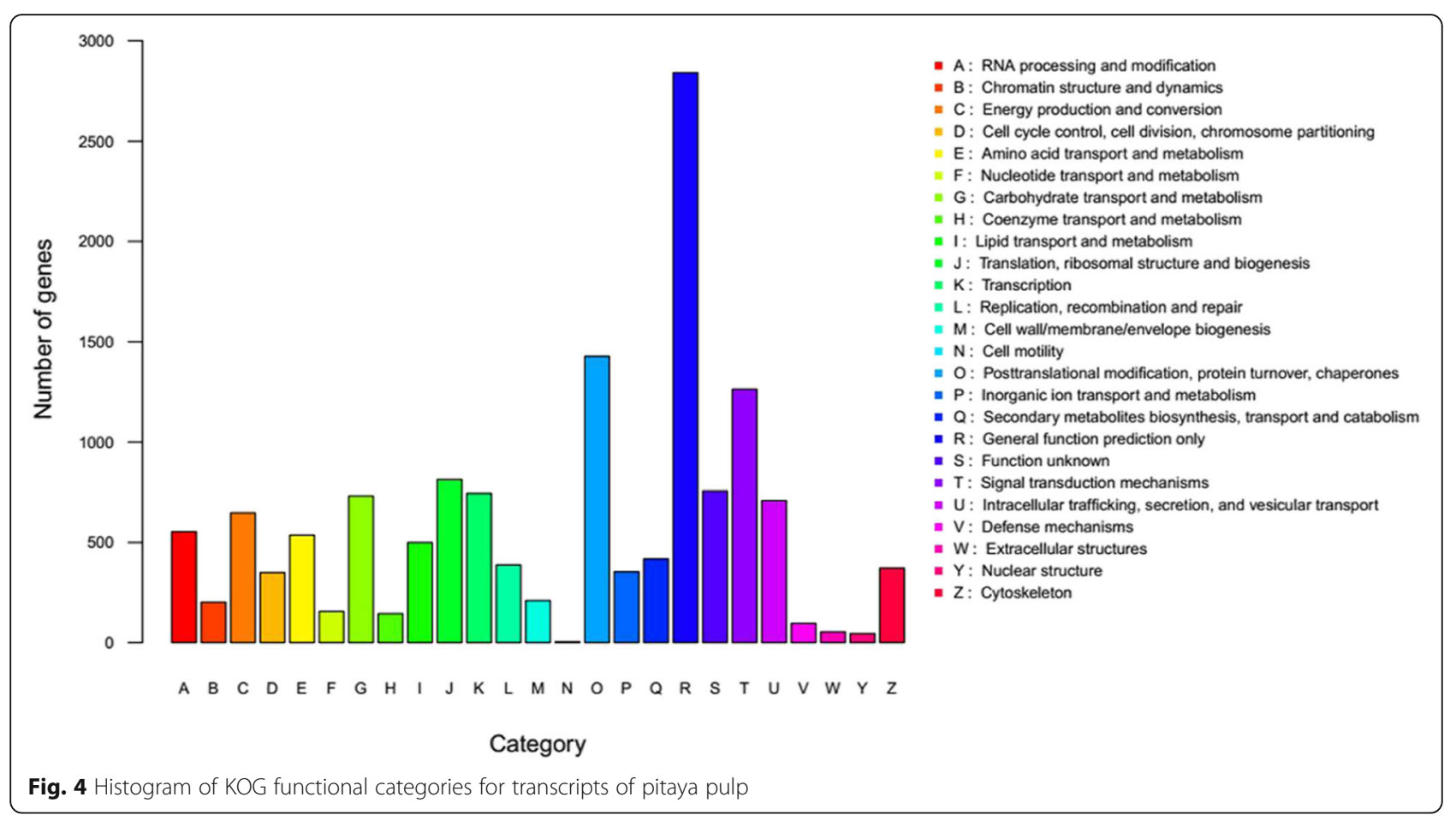

30 differentially expressed miRNAs showed different expression levels in those pitaya tissues. Hmo-miR172a, Hmo-miR394, Hmo-miR530, Hmo-miR6020, HmomiR397b, Hmo-miR160b and Hmo-miR398b displayed similar expression patterns. Hmo-miR156 preferentially expressed in pitaya roots and fruits. Hmo-miR398a strongly expressed in pitaya stamens compared to weak expression in the other tissues. Higher expression levels of Hmo-novel-7 and Hmo-miR171d were detected in petals but moderately or weakly expressed in the other tissues. Hmo-miR164a and Hmo-miR164b displayed constitutive expression and the highest expression level was detected in pitaya fruits. Hmo-miR396b and HmomiR5072 had higher expression in petals of 'Guanhuabai' pitaya compared to higher expression of Hmo-miR171c and Hmo-novel-2 in petals of 'Guanhuahong' pitaya. Hmo-novel-12, Hmo-novel-21, Hmo-novel-15, HmomiR393, Hmo-miR390b, Hmo-miR159a and Hmo-
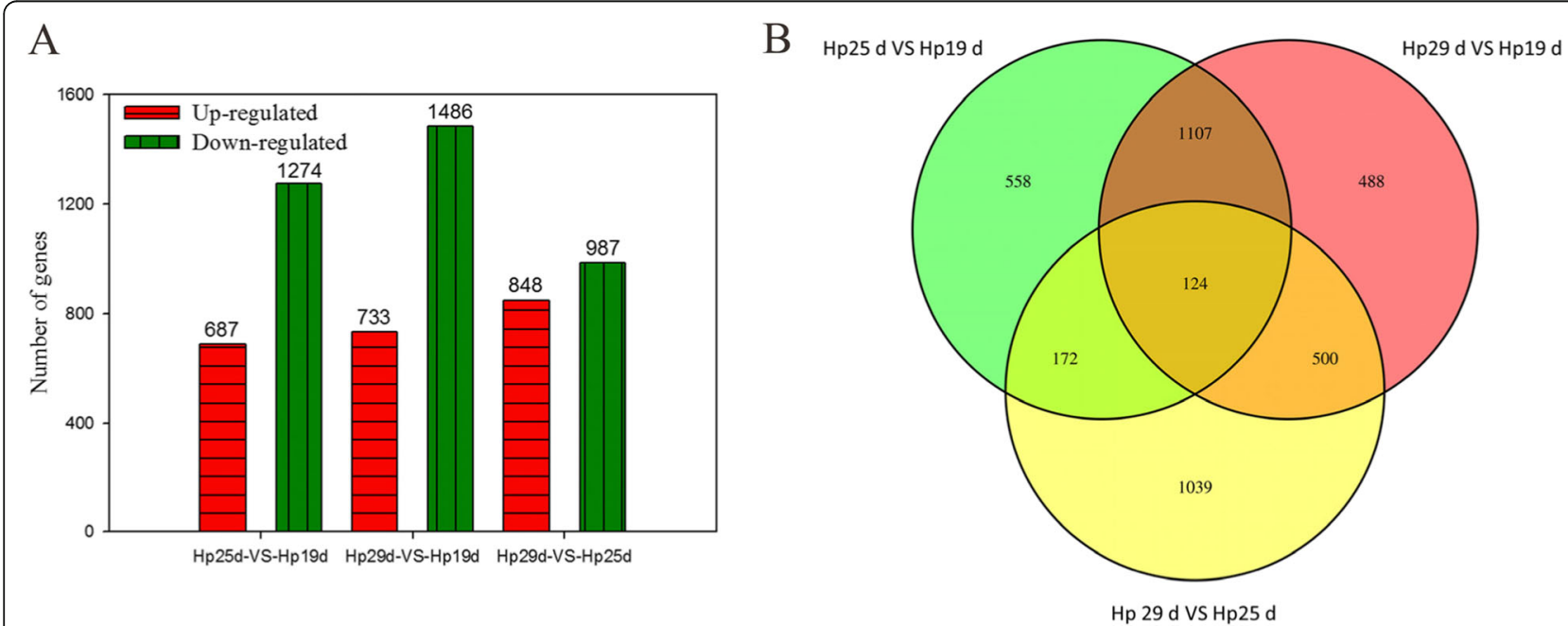

Fig. 5 Differential gene expression profiles based on the libraries of the three coloration stages. a The numbers of up- and down-regulated genes in comparisons of the Hp25 d-VS-Hp19 d, Hp29 d-VS-Hp19 d and Hp29 d-VS-Hp25 d. b The comparison of DEGs between any two stages of the pitaya pulp 

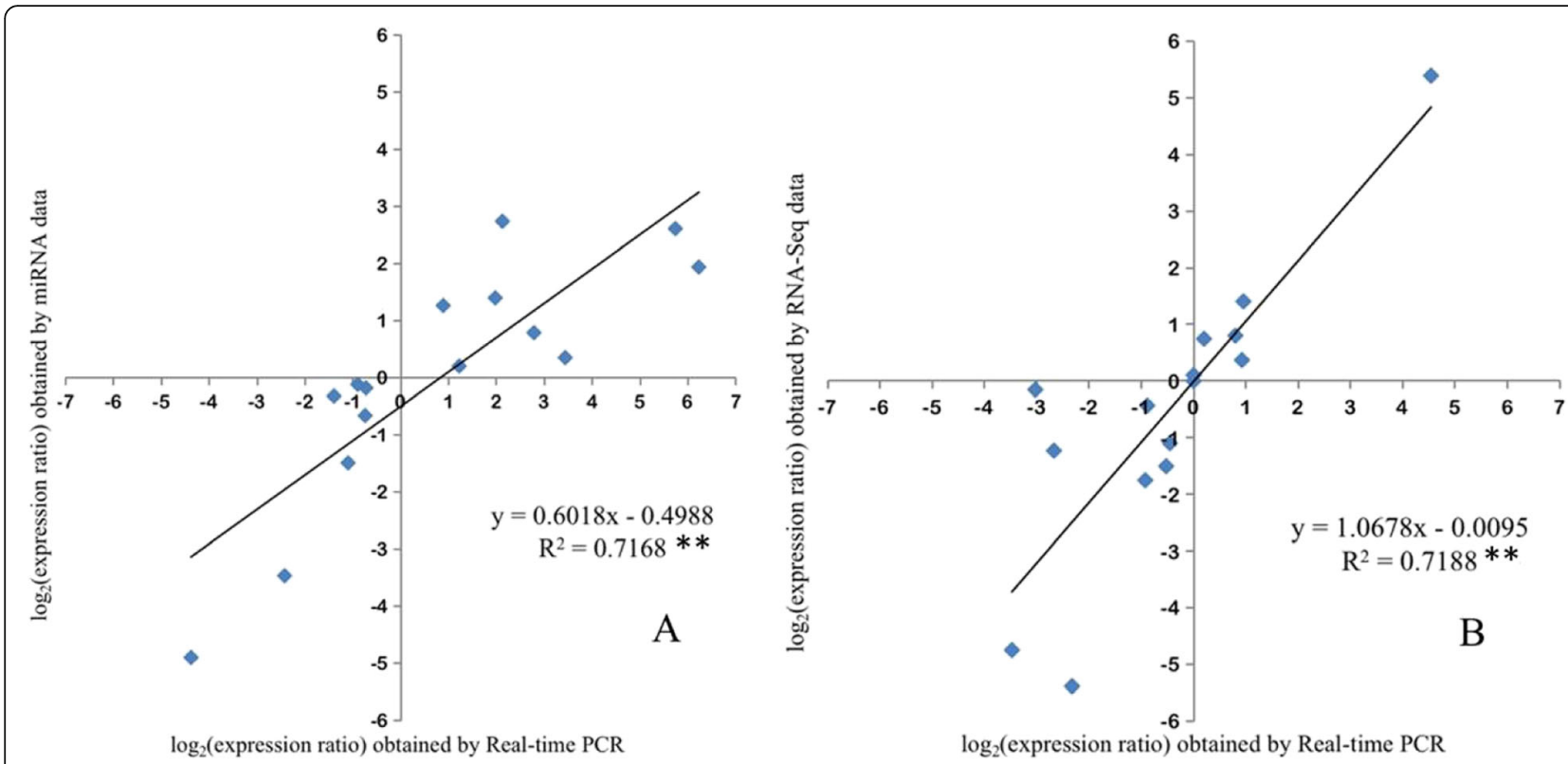

Fig. 6 Verification of the accuracy of the sRNAome and RNA-Seq data by coefficient analyses. a Coefficient analyses of miRNA expression levels from sRNAome and RT-qPCR data. $\mathbf{b}$ Coefficient analyses of gene expression levels from RNA-Seq and RT-qPCR data. The RT-qPCR log 2 values ( $x$ axis) were plotted against high-throughput data (y-axis). ${ }^{* *}$ indicates a significant difference at $p<0.01(n=15)$

miR408 were preferentially expressed in petals and receptacles of 'Guanhuahong' pitaya. Hmo-miR157b and Hmo-miR6300 were highly expressed in 'Guanhuahong' and 'Guanhuabai' pitayas, respectively. Results from RTqPCR indicated that expression of the 30 miRNAs from pitaya had tissue- and/or cultivar- characteristics.

\section{Validation of miRNAs and target genes related to betalain biosynthesis}

miRNAs involved in pitaya betalain biosynthesis were screened based on annotated transcripts and previous reports related to pigment synthesis. Fourteen target genes predicted from 11 miRNAs were assayed by RT-qPCR. The target genes from the 'Guanhuahong' and 'Guanhuabai' pitayas had the same sequences (Figure S5). Sixteen targets showed decreased or increased expression trends along with the increased or decreased expression of the miRNA, suggesting that they might be actively cleaved by miRNAs (Fig. 8). For example, Hpcyt P450-like3 (Fig. 8A9 and B9) and Hpcyt P450-like2 (Fig. 8A10 and B10) targeted by Hmo-miR160a, HmMYB12-like (Fig. 8A5), HmMYBC1-like (Fig. 8A6) and HmMYB2-like (Fig. 8A7) targeted by HmomiR858, and HmCYP71A8-like (Fig. 8A2) targeted by Hmo-miR6020 showed increasing expression trend at first and decreasing thereafter while Hmo-miR160a, HmomiR858 and Hmo-miR6020 showed a reverse trend at all pulp coloration stages of pitaya. HmSPL6-like (Fig. 8A15) targeted by Hmo-miR156 showed decreasing while HmomiR156 showed increasing at all pulp coloration stages of pitaya. However, some targeted genes and their miRNAs such as HmTPST-like (Fig. 8B11) targeted by Hmo-novel15 in 'Guanhuahong' pitaya and HmSPL6-like (Fig. 8A13) targeted by Hmo-miR529b in 'Guanhuabai' pitaya had similar expression patterns at all pulp stages of pitaya.

5 'RACE analyses were further verified the fourteen candidate targets. The HmSPL6-like, HmTT2-like, HmMYB12like, HmMYBC1-like, Hpcyt P450-like3, HmCYP83B1-like and HmTPST-like were confirmed to be cleaved by their corresponding miRNAs (Fig. 9). The cleavage sites of Hmonovel-2 on HmCYP83B1-like and Hmo-novel-15 on HmTPST-like were both occurred at the $10^{\text {th }}$ nucleotide from the $5^{\prime}$-end of miRNAs in the binding region. The cleavage frequency of Hmo-novel-2 on HmCYP83B1-like and Hmo-novel-15 on HmTPST-like was up to 10/10 both in 'Guanhuabai' and 'Guanhuahong' pitayas, respectively. Those results confirmed that Hmo-novel-2 and Hmonovel-15 can guide the cleavage of the mRNA of HmCYP83B1-like and HmTPST-like, respectively. Cleavage occurred mostly at the $10^{\text {th }}$ nucleotide from the $5^{\prime}$-end in the binding sites. However, HmMYBC1-like occurred at the $9^{\text {th }}$ nucleotide in 'Guanhuahong' pitaya, which may be due to the wide range of miRNA cutting mRNA caused by siRNA interference. Besides, the same gene from 'Guanhuahong' pitaya is different from 'Guanhuabai' pitaya (Fig. 9), which may be responsible for the different pulp colors of the two pitaya cultivars.

The relationship between miRNAs and their target genes The transient expression system was constructed to confirm that miRNAs degrade their target genes in vivo. 


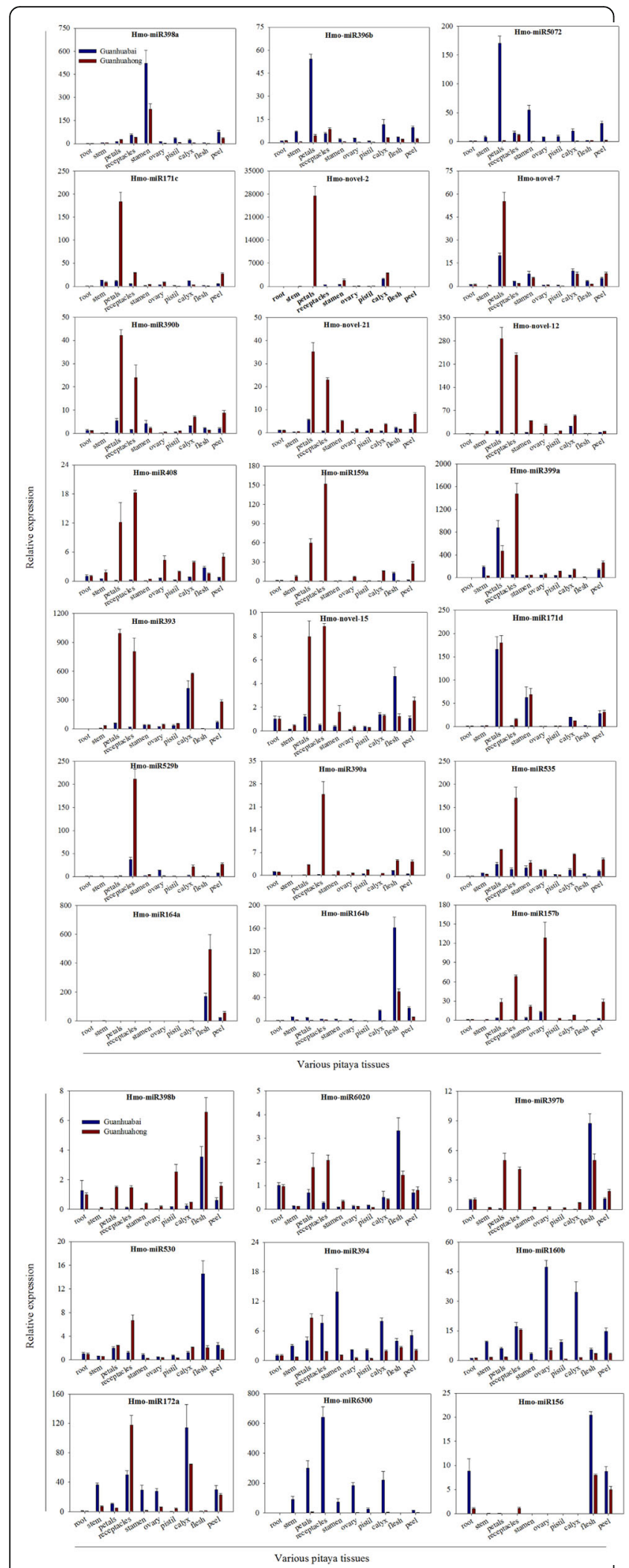

Fig. 7 RT-qPCR analyses of miRNAs in various pitaya tissues using the expression of the $U 6$ gene as the reference
Target sites of miRNAs in target genes and a modified target site (inactivated target site) were inserted into the overexpression vector harboring an enhanced green fluorescent protein $(e G F P)$ gene, respectively. A total of five miRNAs and seven corresponding target genes i.e. Hmo-miR160aHpcyt P450-like2, Hmo-miR6020-HmCYP71A8-like, Hmonovel-2-HmCYP83B1-like, Hmo-novel-15-HmTPST-like, Hmo-miR858-HmMYB12-like, Hmo-miR858-HmMYBC1like and Hmo-miR858-HmMYB2-like were verified their interactions using a tobacco transient expression system (Figure S6). Co-expression of Hmo-miR160a-Hpcyt P450-like2, Hmo-miR6020-HmCYP71A8-like, Hmo-novel-2-HmCYP83 B1-like, Hmo-novel-15-HmTPST-like, Hmo-miR858-HmM YB12-like, Hmo-miR858-HmMYBC1-like and Hmo-miR8 58-HmMYB2-like inhibited the expression of eGFP, indicating that miRNAs degrade their target genes in vivo (Fig. 10B2, 4, 6, 8, 10, 12 and 14). The result was consistent with the positive control (Figure S6B10, 18, 28, 32, 40 and 48). The interactions between miRNAs and target genes could affect miRNA processing resulting in higher precursor accumulation and reduced mature miRNA in pitaya, and further regulate betalain biosynthesis in pitaya (Fig. 10).

\section{Discussion}

Great progress has been made in pitaya betalain studies in term of physical and chemical properties [47, 48], purification and identification [49-54], antioxidant and radical scavenging capacity [55-57] as well as metabolic and transcriptional analyses $[45,46]$. However, no information is available about miRNAs involved in betalain biosynthesis of pitaya. Therefore, identification of pitaya miRNAs associated with their target genes will help our understanding of molecular regulatory mechanisms of pitaya betalain biosynthesis. In this study, transcriptome and sRNAome were performed to explore the role of miRNAs in pitaya coloration mechanisms at different developmental stages. The percentage of $24 \mathrm{nt}$ sRNAs (an average of 58.03\%) was much higher than that of 21 nt sRNAs (an average of 12.16\%) in pitaya pulp (Figure S1). The result was consistent with the length distribution of sRNAs in apple and litchi $[15,20,58]$, but was inconsistent with the findings in strawberry, orange, Brassica juncea, and apple [21, 59-61] suggesting that different plant species have different length sRNA distribution. A total of 95 known miRNAs belonging to 53 miRNA families and 41 new miRNAs were identified from 'Guanhuahong' pitaya ( $H$. monacanthus) (Table S2). miRNAs showed different expression levels during fruit developmental stages of pitaya, indicating that miRNAs play essential roles in pitaya fruit development. Those results suggested that miRNAs are involved in pitaya growth and development through different expressions in various pitaya tissues. 


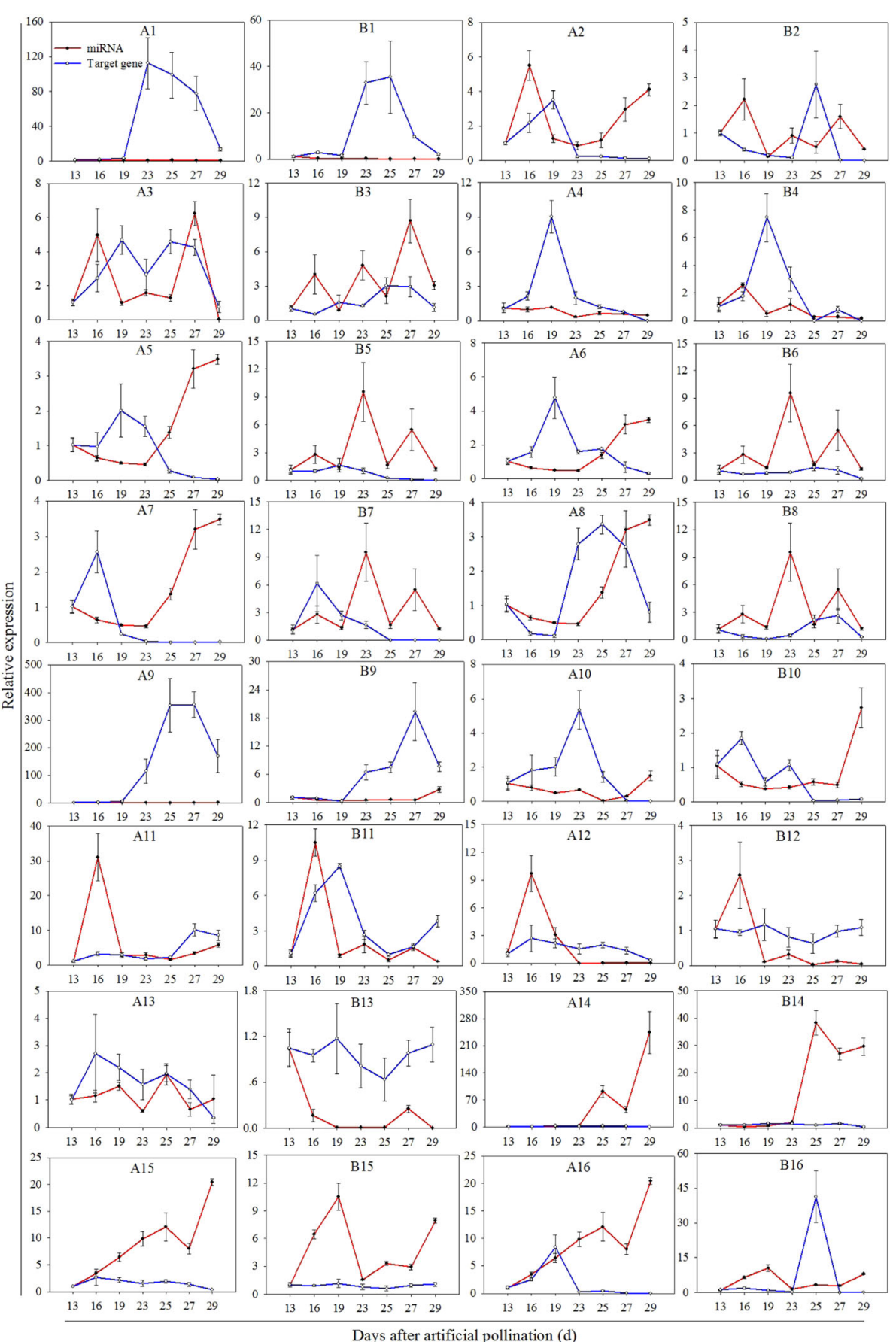

Fig. 8 Expression analyses of miRNAs and their target genes by RT-qPCR. (A1-A16) 'Guanhuabai' pitaya. (B1-B16) 'Guanhuahong' pitaya. (A1-B1) Hmo-miR828a and HmTT2-like. (A2-B2) Hmo-miR6020 and HmCYP71A8-like. (A3-B3) Hmo-novel-2 and HmCYP83B1-like. (A4-B4) Hmo-miR159c and HmGmSGT2-like. (A5-B5) Hmo-miR858 and HmMYB12-like. (A6-B6) Hmo-miR858 and HmMYBC1-like. (A7-B7) Hmo-miR858 and HmMYB2-like. (A8B8) Hmo-miR858 and HmMYB315-like. (A9-B9) Hmo-miR160a and Hpcyt P450-like3. (A10-B10) Hmo-miR160a and Hpcyt P450-like2. (A11-B11) Hmo-novel-15 and HmTPST-like. (A12-B12) Hmo-miR157b and HmSPL6-like. (A13-B13) Hmo-miR529b and HmSPL6-like. (A14-B14) Hmo-miR164a and HmWDTC1-like. (A15-B15) Hmo-miR156 and HmSPL6-like. (A16-B16) Hmo-miR156 and HmSPL16-like

Generally, conserved miRNAs have the same or homologous targets as other plant species, and most of them show a similar function. MIR156 plays a key role in the biosynthesis of secondary metabolites. MIR156 can positively regulate anthocyanin biosynthesis by SPL TFs, while SPL TFs negatively regulate anthocyanin accumulation in Arabidopsis, apple, litchi, and Pyrus pyrifolia [13, 20, 21, 23]. Bgy-mir156 regulates the target gene 


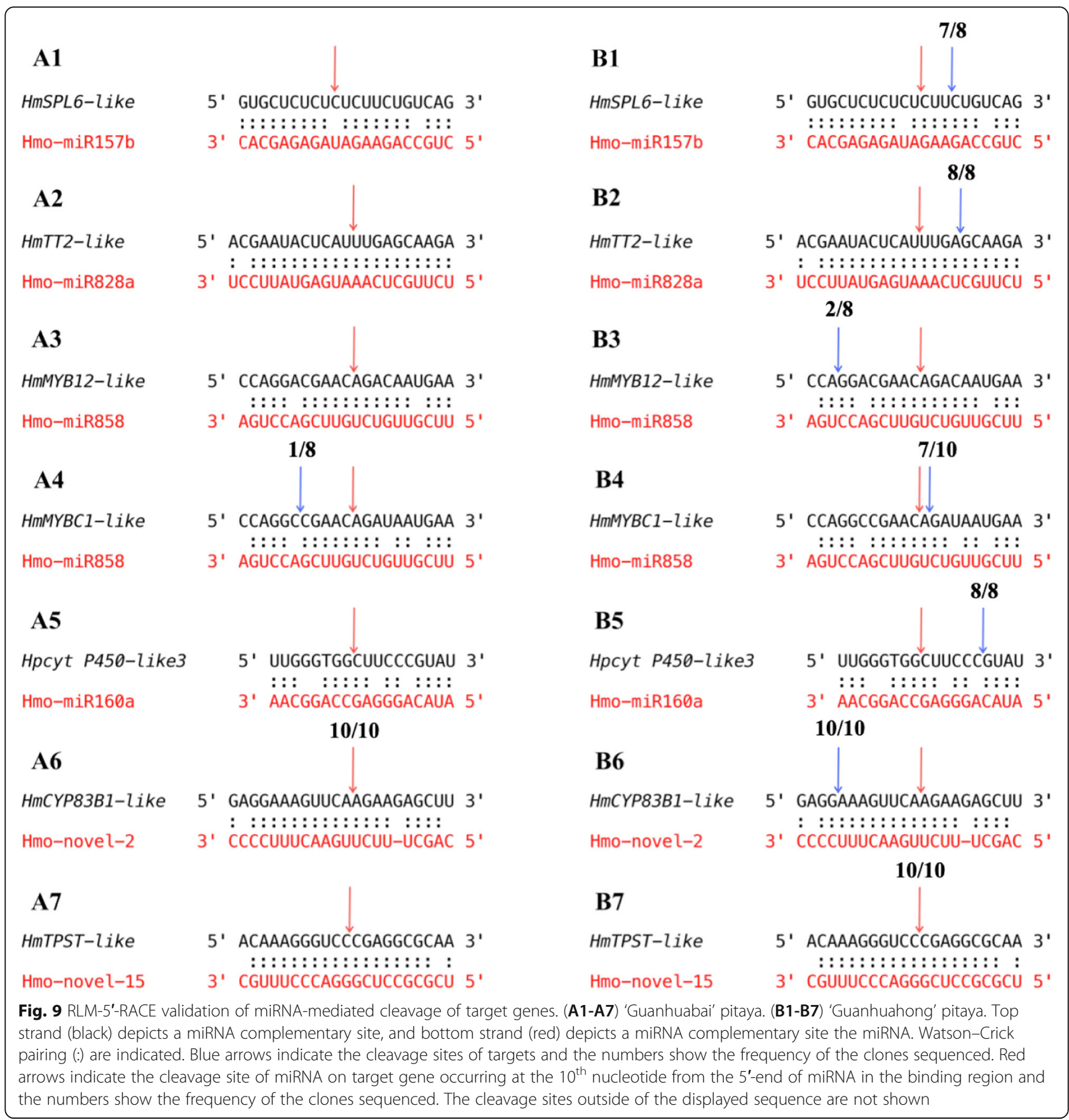

phytoene synthase (PSY) and affects the accumulation of carotenoids in carrot (Daucus carota) [12]. Betalains are secondary metabolites but cannot co-exist naturally in one plant at the same time [62]. Pitaya is a high-value, functional fruit containing abundant betalains. In our study, the highest expression level of Hmo-miR157b was detected on the $23^{\text {rd }}$ DAF (color conversion) in 'Guanhuahong' pitaya, suggesting that Hmo-miR157b may play significant roles in betalain accumulation. Results from 5'RACE showed that HmSPL6-like was targeted by Hmo-miR157b (Fig. 7). Those results indicated that
Hmo-miR157b might positively regulate betalain biosynthesis by SPL TFs, while SPL TFs negatively regulates betalain accumulation in pitaya.

MiR828 participated in anthocyanin biosynthesis by repressing the expression of MYB TFs in Arabidopsis, apple and potato $[14,15,21,24]$. In this study, the expression levels of HmTT2-like after $23^{\text {rd }}$ DAF of 'Guanhuahong' pitaya were lower than that of the 'Guanhuabai' pitaya (Fig. 8A1 and B1). HmTT2-like showed a negative correlation with betalain accumulation in pitaya [45]. Hmo-miR828a was highly active on 

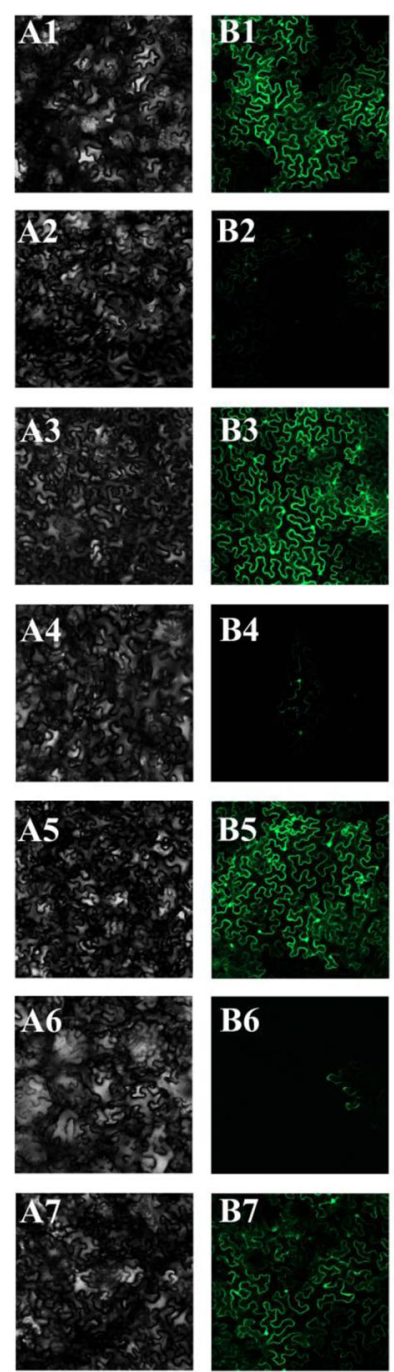
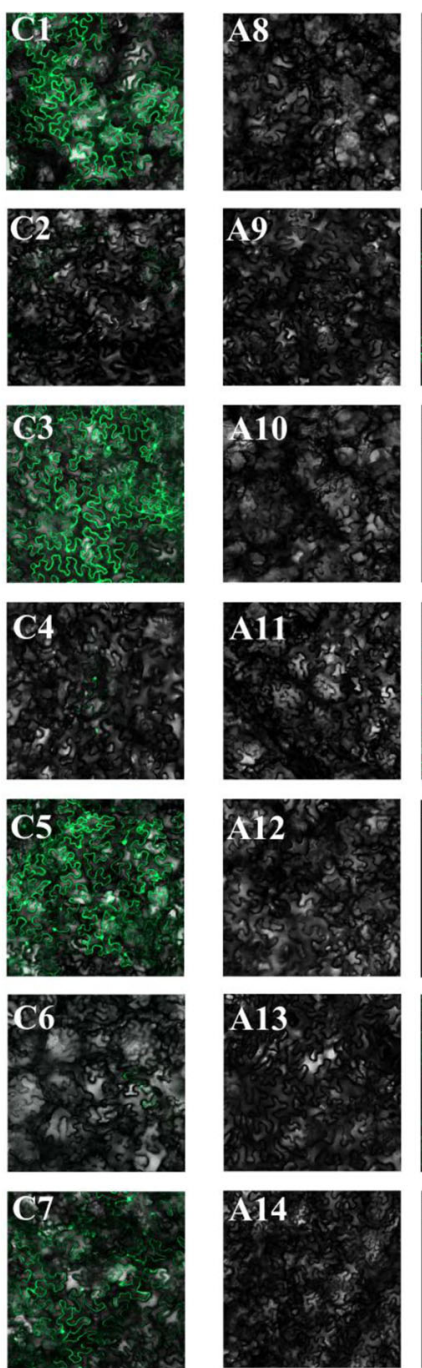
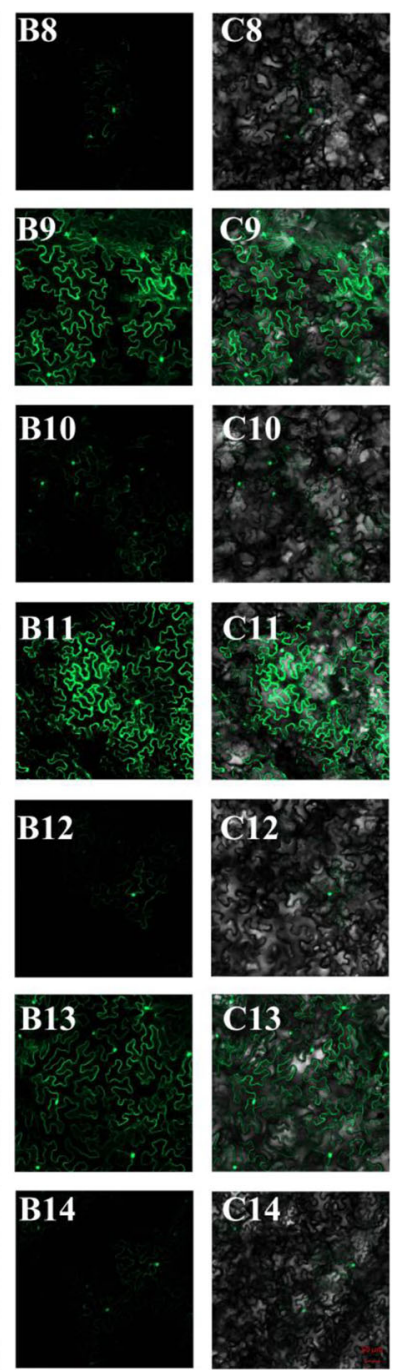

Fig. 10 Transient expressions of miRNAs and their target genes (part). (A1-A14) bright field. (B1-B14) eGFP. (C1-C14) merge. (A1-C1) 35 s::HmCYP71A8-like::.eGFP. (A2-C2) 35 s::HmCYP71A8-like::eGFP+ 35 s:::Pre-Hmo-miR6020. (A3-C3) 35 s::HmMYB12-like::.eGFP. (A4-C4) 35 s::HmMYB12like::.eGFP+ 35 s:::Pre-Hmo-miR858. (A5-C5) 35 s::HmMYBC1-like::eGFP. (A6-C6) 35 s::HmMYBC1-like::eGFP+ 35 s::Pre-Hmo-miR858. (A7-C7) 35 s::HmMYB2-like::eGFP. (A8-C8) 35 s::HmMYB2-like::eGFP+ 35 s::Pre-Hmo-miR858. (A9-C9) 35 s::Hpcyt P450-like2::.eGFP. (A10-C10) 35 s:.Hpcyt P450like2::.eGFP+ 35 s::.Pre-Hmo-miR160a. (A11-C11) 35 s::.HmCYP83B1-like::.eGFP. (A12-C12) 35 s.:.HmCYP83B1-like::eGFP+ 35 s.:.Pre-Hmo-novel-2. (A13-C13) 35 s.:HmTPST-like:.:eGFP. (A14-C14) 35 s.:HmTPST-like:.:eGFP+ 35 s.:Pre-Hmo-novel-15. All transient expressions (including negative and positive controls) are presented in Figure 56

the $23^{\text {rd }}$ DAF of 'Guanhuahong' pitaya (color conversion). Hmo-miR828a could target HmTT2-like (Fig. 9B2), a MYB TF, suggesting that Hmo-miR828a positively regulate betalain accumulation in pitaya. miR858 could directly or indirectly control anthocyanin biosynthesis in Arabidopsis, cotton, apple and tomato, and negatively regulate anthocyanin accumulation by the MYB TF [14, 15, 17, 18]. In the present study, four target genes i.e., HmMYB315-like, HmMYB12-like, HmMYBC1-like and $H m M Y B 2-l i k e$ shared the same negative expression pattern with Hmo-miR858 in 'Guanhuabai' pitaya (Fig. 8A5A8, and B5-B8). 5'RACE and transient expression analyses showed that Hmo-miR858 targeted HmMYB12-like,
HmMYBC1-like, and HmMYB2-like in 'Guanhuahong' pitaya (Fig. 9B3-B4 and Fig. 10A3-A8, B3-B8 and C3-C8). Those results suggested that Hmo-miR858 can promote pitaya betalain accumulation by $M Y B$ genes.

Hpcyt P450-like3 is involved in betalain biosynthesis in H. monacanthus [45]. In this study, Hpcyt P450-like3 was targeted by Hmo-miR160a, suggesting that HmomiR160a was involved in betalain biosynthesis (Fig. 9B5). Novel miRNAs are involved in accumulations of chlorophyll, carotenoid, and anthocyanin. Ttu-novel-48 could regulate chlorophyll accumulation in leaves of durum wheat [6]. Csi-novel-03 regulates carotenoid pathways by AP2 TFs in Citrus [8]. In strawberry receptacle fruit 
ripening, ABA-induced Fa_novel23 resulted in the rapid accumulation of fruit anthocyanin [25]. In our study, two novel miRNAs: Hmo-novel-2 and Hmo-novel-15 were obtained. Hmo-novel-2 influenced betalain accumulation via the regulation of HmCYP83B1-like (Fig. 9A6-B6 and Fig. 10A11-C11 and A12-C12). Hmo-novel-15 was identified as a regulator of betalain biosynthesis regulating the expression of HmTPST-like (Figs. 9B7 and 10A13-C13 and A14-C14). Hmo-miR6020-HmCYP71A8-like was not verified in 5'RACE but in the tobacco transient expression system (Fig. 10A1-C1 and A2-C2) suggesting that the translation inhibited the regulation modes of miRNAs on their targets rather than the degradation of mRNAs. The rest of the differentially expressed miRNAs and their targets in HTS were not confirmed in 5'RACE and tobacco transient expression system, indicating that these genes might not be targets of these miRNAs. Further work is necessary to elucidate their roles in betalain biosynthesis of pitaya.

\section{Conclusions}

In this study, sRNAome and RNA-Seq were first used to identify differentially expressed miRNAs and their target genes involved in betalain biosynthesis. Comprehensive sRNAome analyses uncovered 126 conserved miRNAs and 41 novel miRNAs were obtained from 'Guanhuahong' pitaya (H. monacanthus), among which 95 conserved miRNAs belonged to 53 miRNA families. $26.79 \mathrm{~Gb}$ raw RNASeq data were generated and de novo assembled into 68, 505 transcripts, in which 39,737 were annotated. miRNAs and their target genes involved in betalain accumulation were compared at different developmental stages of pitaya fruit. Seven target genes were verified by $5^{\prime}$ RACE and a tobacco transient expression system. Those Hmo-miRNAs negatively regulated expression of their target mRNAs through guiding corresponding target mRNA cleavage or inhibiting the translation. Hmo-miR157b-HmSPL6-like, Hmo-miR160a-Hpcyt P450-like3, Hmo-miR6020-HmCYP 71A8-like, Hmo-novel-2-HmCYP83B1-like, Hmo-novel-15HmTPST-like, Hmo-miR828a-HmTT2-like, Hmo-miR858HmMYB12-like, Hmo-miR858-HmMYBC1-like and HmomiR858-HmMYB2-like are possibly involved in betalain biosynthesis in pitaya. The present study provides new information that miRNAs are actively involved in betalain accumulation of pitaya fruit by regulating the upstream TFs, which may contribute to a further understanding of miRNAs in betalain biosynthesis of pitaya.

\section{Methods}

\section{Plant materials}

Two pitaya cultivars, i.e., 'Guanhuahong' (red peel with red pulp, H. monacanthus) and 'Guanhuabai' (red peel with white pulp, $H$. undatus) and Nicotiana benthamiana were used as plant materials. 'Guanhuahong' and 'Guanhuabai' pitayas, authenticated by Professor Guibing
$\mathrm{Hu}$ and Yonghua Qin (College of Horticulture, South China Agricultural University), were selected from 860 seedlings of 'Hongshuijing' (H. monacanthus) [63-65]. Nicotiana benthamiana was grown in a greenhouse with a condition of $16 \mathrm{~h} / 8 \mathrm{~h}$ day/night at $25^{\circ} \mathrm{C}$ and was used for interactions between miRNAs and their target gene assays in vivo. The South China Agricultural University provided all plant materials used in this study, and no specific permissions were required for the collection of those samples for research purposes following institutional, national and international guidelines. Fruits of 'Guanhuahong' and 'Guanhuabai' pitayas from the same orchard of Dalingshan Forest Park were separated into peels and pulps on the $13^{\text {th }}, 16^{\text {th }}, 19^{\text {th }}, 23^{\text {rd }}, 25^{\text {th }}, 27^{\text {th }}$ and $29^{\text {th }}$ DAF (Figure S7) for expression analyses of crucial miRNAs and their targets. Pulps from 'Guanhuahong' pitaya on the $19^{\text {th }}$ DAF (white pulp stage, Hp19d, Hp19d_1, and Hp19d_2), 25 ${ }^{\text {th }}$ DAF (pulp coloration stages, Hp25d, Hp25d_1, and Hp25d_2) and $29^{\text {th }}$ DAF (mature stage, Hp29d, Hp29d_1, and Hp29d_2) were used for RNA-Seq and sRNAome. All samples were frozen immediately in liquid nitrogen and stored at $-80^{\circ} \mathrm{C}$ until use.

\section{SRNAome and RNA-Seq}

Total RNA was extracted using the TruSeq Small RNA Sample Prep Kits (Illumina, San Diego, USA) according to the manufacturer's instructions. RNA-Seq and sRNAome libraries were performed according to the procedures of Han et al. (2016) and Liu et al. (2016), respectively $[20,66]$. Six small RNA libraries (Hp19d_1, Hp19d_2, Hp25d_1, Hp25d_2, Hp29d_1 and Hp29d_2) from two biological replicates and three RNA-Seq libraries (Hp19d, Hp25d and Hp29d) were constructed (https://dataview.ncbi.nlm.nih.gov/object/PRJNA588519? reviewer=ko91rr55muepqo1d25plnp0kv4). All HTS was performed by LC-BIO (Hangzhou, China).

\section{Bioinformatic analyses}

Clean sRNA sequences were obtained from sRNAome raw data (raw reads) by removing adapters, low-quality tags, and contaminants. Clean sRNA sequences were compared with the Rfam database (http://rfam.sanger.ac.uk/) after removing rRNA, tRNA, snRNA, and snoRNA. To screen known miRNAs, the clean data were mapped to the reference sequence in miRBase21.0 by Bowtie [67]. miRNA precursor was submitted to RNAfold software (http://rna.tbi.univie.ac.at/cgi-bin/RNAfold.cgi) to identify novel miRNA [44].

Analyses of transcriptome raw data was identical to that of sRNAome raw data. Clean transcriptome data were assembled into non-redundant unigenes using Trinity (http://trinityrnaseq.github.io/). Unigenes were 
tentatively identified based on the best hits against known sequences in the database.

\section{Prediction and functional annotation of target gene}

Target genes of differentially-expressed miRNAs were predicted by Target Finder software. GO, KOG and KEGG were used to analyze the functions of target genes. $E$-value $\leq 10^{-5}$ was considered as significant enrichment.

\section{Sequence alignment analyses}

Multiple sequences were aligned using DNAMAN software (version 8). The miRNA target sites were identified using sequence alignments and manual analyses.

\section{Analyses of miRNAs and target genes by RT-qPCR}

Stem-loop RT-qPCR was used to confirm expression of miRNAs since it is a highly sensitive method for detection of miRNAs [68]. cDNAs were produced from $1.0 \mu \mathrm{g}$ of total RNA samples using the MMLV-reverse transcriptase (Invitrogen) with miRNA specific stem-loop and oligo(dT) primers, respectively. The specific primers and PCR reactions were performed according to our previous method [20]. RT-qPCR was conducted in ABI 7500 real-time PCR System (Applied Biosystems, CA, USA) using the SYBR qPCR Mix (Vazyme). Twenty microliters reaction mixture contained $2.0 \mu \mathrm{L}$ of diluted cDNAs $(\sim 15 \mathrm{ng} / \mu \mathrm{L}), 10.0 \mu \mathrm{L}$ $2 \times$ SYBR qPCR Mix (Vazyme), $0.5 \mu \mathrm{L}$ of each primer $(10 \mu \mathrm{M})$ and $7.0 \mu \mathrm{L} d \mathrm{ddH}_{2} \mathrm{O}$. All experiments were performed in triplicate. $U 6$ and actin gene were used as reference genes $[69,70]$. The sequences of miRNAs and target genes were shown in Tables S2 and S10, respectively. All primers used for RT-qPCR analyses were listed in Tables S4, S5 and S6. The expression levels of miRNAs and target genes were calculated by $2^{-\triangle \Delta C}$ T method [71].

\section{5'RACE analyses}

To verify the miRNA-mediated cleavage events, RNA ligase-mediated 5'RACE (RLM-RACE) was performed using the SMARTer RACE 5'/3'Kit User Manual (012615) (TaKaRa, Dalian, China) according to the manufacturer's manual. $1.0 \mu \mathrm{g}$ total RNA from the pulps of the $29^{\text {th }}$ DAF 'Guanhuahong' and 'Guanhuabai' pitayas was ligated with 5'RNA adapters, respectively. The ligated mRNA was reversely transcribed by oligo (dT) primer. $5^{\prime}$ end products were obtained using $5^{\prime}$ adaptor primers and 3 ' gene-specific primers. PCR products were inserted into pMD18-T vector (TaKaRa). Specific primers used for nested PCR were shown in Table S7.

\section{Transient expression analysis}

A transient expression system was used to confirm the interaction between miRNAs and their target genes in vivo. Construction of expression vectors were constructed following the procedure of Liu et al. [69]. Over- expression vectors of miRNAs related to the betalain biosynthesis and a control miRNA were constructed respectively. Co-expression of miRNAs and their targets in $N$. benthamiana leaves using Agrobacterium tumefaciens GV3101 infiltration. Transient expression in N. benthamiana was performed as described by Sparkes et al. (2006) [72]. Three days after infiltration, leaves were observed with a laser scanning confocal microscope (Zeiss LSM800) according to the following parameters: laser wavelength of $488 \mathrm{~nm}$ (laser intensity of $0.2 \%$ ), master gain values ranging from 550 to $700 \mathrm{~V}$, ESID gain value of 3 and digital gain value of 1.0. The transient expression assays were repeated at least three times.

\section{Supplementary information}

Supplementary information accompanies this paper at https://doi.org/10. 1186/s12870-020-02622-x.

Additional file 1: Figure S1. The length distributions of miRNA fragments in pitaya (unique).

Additional file 2: Figure S2. The length distributions of assembled transcripts and genes. A, The length distributions of transcripts; $\mathbf{B}$, The length distributions of genes.

Additional file 3: Figure S3. GC content distributions of assembled transcripts and genes. A, GC content distributions of transcripts; B, GC content distributions of genes.

Additional file 4: Figure S4. Number of miRNA family members in pitaya pulp.

Additional file 5: Figure S5. The sequences of 17 target genes from the two pitaya cultivars. '-W', 'Guanhuabai' pitaya. '-R', 'Guanhuahong' pitaya. Red lines indicate sequence of target genes primers for real-time PCR. Green lines indicate outer specific primers for nested PCR. Blue lines indicate inner specific primers for nested PCR.

Additional file 6: Figure S6. Transient expressions of miRNAs and their target genes (all). A1-A49, bright field; B1-B49, eGFP; C1-C49, merge; A1C1, 35 s:::eGFP; A2-C2, WT; A3-C3, 35 s::Pre-miR164; A4-C4, 35 s::Pre-HmomiR6020; A5-C5, 35 s.:HmCYP71A8-like:.:eGFP; A6-C6, 35 s.:positive control HmCYP71A8-like::eGFP; A7-C7, 35 s:.:negative control HmCYP71A8-like::eGFP; A8-C8, 35 s::HmCYP71A8-like::eGFP+ 35 s.:.Pre-miR164; A9-C9, 35

s::HmCYP71A8-like::eGFP+ 35 s::Pre-Hmo-miR6020; A10-C10, 35 s::positive control HmCYP71A8-like::.eGFP+ 35 s.:Pre-Hmo-miR6020; A11-C11, 35 s::negative control HmCYP71A8-like::eGFP+ 35 s::.Pre-Hmo-miR6020; A12C12, 35 s.:Pre-Hmo-miR858; A13-C13, 35 s.:HmMYB12-like::.eGFP; A14-C14, 35 s::positive control HmMYB12-like::eGFP; A15-C15, 35 s::negative control HmMYB12-like::eGFP; A16-C16, 35 s.:.HmMYB12-like::.eGFP+ 35 s.:Pre-miR164; A17-C17, 35 s::.HmMYB12-like::.eGFP+ 35 s.:.Pre-Hmo-miR858; A18-C18, 35 s:::positive control HmMYB12-like:.:eGFP+ 35 s::Pre-Hmo-miR858; A19-C19, 35 s::negative control HmMYB12-like::.eGFP+ 35 s::Pre-Hmo-miR858; A20C20, 35 s:.:HmMYBC1-like:.:eGFP; A21-C21, 35 s::HmMYBC1-like:.:eGFP+ 35 s::Pre-miR164; A22-C22, 35 s:.:HmMYBC1-like::eGFP+ 35 s::Pre-Hmo-miR858; A23-C23, 35 s::HmMYB2-like::eGFP; A24-C24, 35 s::HmMYB2-like::eGFP+ 35 s.:.Pre-miR164; A25-C25, 35 s::HmMYB2-like::.eGFP+ 35 s.:Pre-Hmo-miR858; A26-C26, 35 s.:Pre-Hmo-miR160a; A27-C27, 35 s::Hpcyt P450-like2::eGFP; A28-C28, 35 s:::positive control Hpcyt P450-like2::eGFP; A29-C29, 35 s::negative control Hpcyt P450-like2::eGFP; A30-C30, 35 s::Hpcyt P450-like2::eGFP+ 35 s.:Pre-miR164; A31-C31, 35 s:.:Hpcyt P450-like2::.eGFP+ 35 s::Pre-HmomiR160a; A32-C32, 35 s:.:positive control Hpcyt P450-like2::eGFP+ 35 s:.:PreHmo-miR160a; A33-C33, 35 s:.:negative control Hpcyt P450-like2::.eGFP+ 35 s::Pre-Hmo-miR160a; A34-C34, 35 s::Pre-Hmo-novel-2; A35-C35, 35 s.:.HmCYP83B1-like.:.eGFP; A36-C36, 35 s:.:positive control HmCYP83B1-like:.:eGFP; A37-C37, 35 s::negative control HmCYP83B1-like::eGFP; A38-C38, 35 s::HmCYP83B1-like::.eGFP+ 35 s.:Pre-miR164; A39-C39, 35 s.:.HmCYP83B1-like:.:eGFP+35 s.:Pre-Hmo-novel-2; A40-C40, 35 s::positive control HmCYP83B1like:.:eGFP+ 35 s.:.Pre-Hmo-novel-2; A41-C41, 35 s:.:negative control 
HmCYP83B1-like::eGFP+ 35 s::Pre-Hmo-novel-2; A42-C42, 35 s::Pre-Hmonovel-15; A43-C43, 35 s::HmTPST-like::eGFP; A44-C44, 35 s.:positive control HmTPST-like::eGFP; A45-C45, 35 s.:negative control HmTPST-like::eGFP; A46C46, 35 s::HmTPST-like::eGFP+ 35 s::Pre-miR164; A47-C47, 35 s.:.HmTPST-like::eGFP+ 35 s::Pre-Hmo-novel-15; A48-C48, 35 s::positive control HmTPSTlike::eGFP+ 35 s::Pre-Hmo-novel-15; A49-C49, 35 s::.negative control HMTPST-like::eGFP+ 35 s::Pre-Hmo-novel-15.

Additional file 7: Figure S7. Different fruit developmental stages of 'Guanhuahong' (A) and 'Guanhuabai' (B) pitayas. A1 and B1, 13 d; A2 and $\mathrm{B} 2,16 \mathrm{~d} ; \mathrm{A} 3$ and $\mathrm{B} 3,19 \mathrm{~d} ; \mathrm{A} 4$ and $\mathrm{B} 4,23 \mathrm{~d} ; \mathrm{A} 5$ and $\mathrm{B} 5,25 \mathrm{~d} ; \mathrm{A} 6$ and $\mathrm{B} 6$, $27 \mathrm{~d} ; \mathrm{A} 7$ and $\mathrm{B} 7,29 \mathrm{~d}$. Bar $=2.0 \mathrm{~cm}$

Additional file 8: Table S1. Statistics of miRNA sequences in pitaya.

Additional file 9: Table S2. The information of miRNAs in pitaya.

Additional file 10: Table S3. Statistics of gene annotation in six databases.

Additional file 11: Table S4. Sequences of miRNA-specific primers for reverse transcription.

Additional file 12: Table S5. Sequences of miRNA primers for real-time PCR

Additional file 13: Table S6. Sequences of target genes primers for real-time PCR.

Additional file 14: Table S7. Sequences of specific primers for $5^{\prime} R A C E$. Additional file 15: Table S8. The information of part RNA-Seq data in pitaya.

Additional file 16: Table S9. Prediction of targets for differentially expression miRNAs in pitaya.

Additional file 17: Table S10. CDNA sequences of 17 target genes.

\section{Abbreviations}

5'RACE: 5' rapid amplification of CDNA ends; AGO: Argonaute; CAM: Crassulacean acid metabolism; CDNA: Complementary DNA; CYP: Cytochrome P450; Cyt P450: Cytochrome P450; DAF: Day after flowering; DEG: Differential expression gene; DOD: Dioxygenase; DOPA: 4,5dihydroxy-phenylalanine; eGFP: Enhanced green fluorescent protein; GO: Gene Ontology; GTs: Glucosyltransferases; HTS: High-throughput sequencing; KEGG: Kyoto Encyclopedia of Genes and Genomes; KOG: euKaryotic Ortholog Groups; miRNA: microRNA; MYB: Myb protooncogene protein; nt: Nucleotide; PSY: Phytoene synthase; RISC: RNAinduced silencing complex; RNA-Seq: RNA sequencing; RPKM: Reads Per Kilobase of exon model per Million mapped read; RT-qPCR: Reverse transcription quantitative real-time polymerase chain reaction; SPL: Squamosa promoter-binding-like; TFs: Transcription factors; TPST: Protein-tyrosine sulfotransferase; TYR: Tyrosinase; WDTC1: WD and tetratricopeptide repeats protein 1

\section{Acknowledgements}

The authors would also like to thank fellow members of the orchard of Dalingshan Forest Park for management of experimental materials.

\section{Authors' contributions}

YHQ and CBC conceived the study, and designed the experiments. CBC, FFX and QZH conducted the experiment. LLZ, ZKZ, RZ, JTZ and GBH analyzed data. CBC, NTZ and YHQ wrote the manuscript. All Authors read and approved the manuscript.

\section{Funding}

This work was supported by the Key Realm R\&D Program of Guangdong Province (2018B020202011), National Natural Science Foundation of China (31972367), Key Science and Technology Planning Project of Guangzhou (201904020015), Science and Technology Program of Guangzhou (202002020060, 201704020003 and 2014Y2-00164), and YangFan Innovative and Entepreneurial Research Team Project (2014YT02H013). The funding organizations paid the experimental costs and publication fees for this research but did not play any role in the design of the study nor in the collection, analysis and interpretation of data, nor in the writing of the manuscript.

\section{Availability of data and materials}

The transcriptome clean raw reads data that support the findings of this study have been submitted to NCBI Sequence Read Archive (SRA) under Accession (SAMN13253272- SAMN13253280), Bioproject: PRJNA588519. All data generated or analyzed during this study are included in this published article and its supplementary information files. The authors are pleased to share with the data upon request.

\section{Ethics approval and consent to participate}

Not applicable.

\section{Consent for publication}

Not applicable.

\section{Competing interests}

The authors declare that they have no conflicts of interest.

\section{Author details}

${ }^{1}$ State Key Laboratory for Conservation and Utilization of Subtropical Agrobioresources/Guangdong Provincial Key Laboratory of Postharvest Science of Fruits and Vegetables/Key Laboratory of Biology and Genetic Improvement of Horticultural Crops (South China), Ministry of Agriculture and Rural Affairs, College of Horticulture, South China Agricultural University, Guangzhou, Guangdong 510642, P. R. China. ${ }^{2}$ French Associates Institute for Agriculture and Biotechnology of Drylands, The J. Blaustein Institutes for Desert Research, Ben-Gurion University of the Negev, Sede Boqer Campus, 84990 Beersheba, Israel.

Received: 3 January 2020 Accepted: 25 August 2020

Published online: 22 September 2020

\section{References}

1. Rogers K, Chen XM. Biogenesis, turnover, and mode of action of plant microRNAs. Plant Cell. 2013;25:2383-99.

2. Liu YL, Ke LL, Wu GZ, Xu YT, Wu XM, Xia R, et al. miR3954 is a trigger of phasiRNAs that affects flowering time in Citrus. Plant J. 2017;92:263-75.

3. Guo Y, Zhao S, Zhu C, Chang X, Yue C, Wang Z. Identification of droughtresponsive miRNAs and physiological characterization of tea plant (Camellia sinensis L.) under drought stress. BMC Plant Biol. 2017;17:211.

4. Feng $\mathrm{H}, \mathrm{Xu}$ M, Zheng X, Zhu TY, Gao XN, Huang LL. MicroRNAs and their targets in apple (Malus domestica cv. "Fuji") involved in response to infection of pathogen Valsa mali. Front Plant Sci. 2017:8:2081.

5. Canto-Pastor A, Molla-Morales A, Ernst E, Dahl W, Zhai J, Yan Y, et al. Efficient transformation and artificial miRNA gene silencing in Lemna minor. Plant Biol. 2015;171:59-65.

6. De Paola D, Zuluaga DL, Sonnante G. The miRNAome of durum wheat: isolation and characterisation of conserved and novel microRNAs and their target genes. BMC Genomics. 2016;17:505

7. Tong AZ, Yuan Q, Wang S, Peng JJ, Lu YW, Zheng HY, et al. Altered accumulation of Osa-miR171b contributes to rice stripe virus infection by regulating disease symptoms. J Exp Bot. 2017;68:4357-67.

8. Xu Q, Liu YL, Zhu AD, Wu XM, Ye JL, Yu KQ, et al. Discovery and comparative profiling of microRNAs in a sweet orange red-flesh mutant and its wild type. BMC Genomics. 2010;11:246.

9. Kim J, Park JH, Lim CJ, Lim JY, Ryu JY, Lee BW, et al. Small RNA and transcriptome deep sequencing proffers insight into floral gene regulation in Rosa cultivars. BMC Genomics. 2012;13:657.

10. Zeng $S H$, Liu YL, Pan LZ, Hayward A, Wang Y. Identification and characterization of miRNAs in ripening fruit of Lycium barbarum $L$ using high-throughput sequencing. Front Plant Sci. 2015;6:778.

11. Koul A, Yogindran S, Sharma D, Kaul S, Rajam MV, Dhar MK. Carotenoid profiling, in silico analysis and transcript profiling of miRNAs targeting carotenoid biosynthetic pathway genes in different developmental tissues of tomato. Plant Physiol Biochem. 2016;108:412-21.

12. Bhan B, Koul A, Sharma D, Manzoor MM, Kaul S, Gupta S, et al. Identification and expression profiling of miRNAs in two color variants of carrot (Daucus carota L.) using deep sequencing. PLoS One. 2019;14:e212746.

13. Gou JY, Felippes FF, Liu CJ, Weigel D, Wang JW. Negative regulation of anthocyanin biosynthesis in arabidopsis by a miR156-targeted SPL transcription factor. Plant Cell. 2011;23:1512-22. 
14. Luo QJ, Mittal A, Jia F, Rock CD. An autoregulatory feedback loop involving PAP1 and TAS4 in response to sugars in Arabidopsis. Plant Mol Biol. 2012;80: $117-29$

15. Xia R, Zhu H, An YQ, Beers EP, Liu ZR. Apple miRNAs and tasiRNAs with novel regulatory networks. Genome Biol. 2012;13:R47.

16. Yan J, Gu YY, Jia XY, Kang WJ, Pan SJ, Tang XQ, et al. Effective small RNA destruction by the expression of a short tandem target mimic in Arabidopsis. Plant Cell. 2012;24:415-27.

17. Guan XY, Pang MX, Nah G, Shi XL, Ye WX, Stelly DM, et al. miR828 and miR858 regulate homoeologous MYB2 gene functions in Arabidopsis trichome and cotton fibre development. Nat Commun. 2014;5:3050.

18. Jia XY, Shen J, Liu H, Li F, Ding N, Gao CY, et al. Small tandem target mimicmediated blockage of microRNA858 induces anthocyanin accumulation in tomato. Planta. 2015;242:283-93.

19. Wang L, Zengj HQ, Song J, Feng SJ, Yang ZM. miRNA778 and SUVH6 are involved in phosphate homeostasis in Arabidopsis. Plant Sci. 2015;238:27385.

20. Liu R, Lai B, Hu B, Qin YH, Hu GB, Zhao JT. Identification of microRNAs and their target genes related to the accumulation of anthocyanins in Litchi chinensis by high-throughput sequencing and degradome analysis. Front Plant Sci. 2016:7:e179.

21. Qu D, Yan F, Meng R, Jiang XB, Yang HJ, Gao ZY, et al. Identification of microRNAs and their targets associated with fruit-bagging and subsequent sunlight re-exposure in the "granny smith" apple exocarp using highthroughput sequencing. Front Plant Sci. 2016;7:27.

22. Roy S, Tripathi AM, Yadav A, Mishra P, Nautiyal CS. Identification and expression analyses of miRNAs from two contrasting flower color cultivars of Canna by deep sequencing. PLoS ONE. 2016;11:e0147499.

23. Qian MJ, Ni JB, Niu QF, Bai SL, Bao L, Li JZ, et al. Response of miR156-SPL module during the red peel coloration of bagging-treated Chinese sand pear (Pyrus pyrifolia Nakai). Front Physiol. 2017;8:550.

24. Bonar N, Liney M, Zhang RX, Austin C, Dessoly J, Davidson D, et al. Potato miR828 is associated with purple tube skin and flesh color. Front Plant Sci. 2018;9:1742

25. Li DD, Mou WS, Xia R, Li L, Zawora C, Ying TJ, et al. Integrated analysis of high-throughput sequencing data shows abscisic acid-responsive genes and miRNAs in strawberry receptacle fruit ripening. Hortic Res. 2019;6:26.

26. Nobel PS, De La Barrera E. $\mathrm{CO}_{2}$ uptake by the cultivated hemiepiphytic cactus, Hylocereus undatus. Ann Appl Biol. 2004;144:1-8.

27. Mizrahi Y, Nerd A, Nobel PS. Cacti as crops. Hortic Rev. 1997;18:291-319.

28. Ortiz-Hernández YD, Carrillo-Salazar JA. Pitaya (Hylocereus spp.): a short review. Comunicata Sci. 2012;3:220-37.

29. Esquivel P, Stintzing FC, Carle R. Comparison of morphological and chemical fruit traits from different pitaya genotypes (Hylocereus sp.) grown in Costa Rica. J Appl Bot Food Qual. 2007:81:7-14

30. Stintzing FC, Carle R. Betalains-emerging prospects for food scientists. Trends Food Sci Technol. 2007:18:514-25.

31. Lee EJ, An D, Nguyen C, Patil BS, Kim J, Yoo KS. Betalain and betaine composition of greenhouse- or field-produced beetroot (Beta vulgaris L.) and inhibition of HepG2 cell proliferation. J. Agric. Food Chem. 2014;62: 1324-31.

32. Suh DH, Lee S, Heo DY, Kim YS, Cho SK, Lee S, et al. Metabolite profiling of red and white pitayas (Hylocereus polyrhizus and Hylocereus undatus) for comparing betalain biosynthesis and antioxidant activity. J Agric Food Chem. 2014:62:8764-71.

33. Timoneda A, Feng T, Sheehan H, Walker-Hale N, Pucker B, Lopez-Nieves S, et al. The evolution of betalain biosynthesis in Caryophyllales. New Phytol. 2019;224:71-85.

34. Adnan L, Osman A, Hamid AA. Antioxidant activity of different extracts of red pitaya (Hylocereus polyrhizus) seed. Int J Food Prop. 2011;14:1171-81.

35. Song H, Zheng Z, Wu J, Lai J, Chu Q, Zheng X. White pitaya (Hylocereus undatus) juice attenuates insulin resistance and hepatic steatosis in dietinduced obese mice. PLoS ONE. 2016;11:e01496702.

36. Solovchenko AE, Merzlyak MN. Screening of visible and UV radiation as a photoprotective mechanism in plants. Russian J Plant Physiol. 2008:55:71937.

37. Jamaludin NA, Ding P, Hamid AA. Physico-chemical and structural changes of red-fleshed dragon fruit (Hylocereus polyrhizus) during fruit development. J Sci Food Agric. 2011;91:278-85.

38. Jain G, Gould KS. Functional significance of betalain biosynthesis in leaves of Disphyma australe under salinity stress. Environ Exp Bot. 2015;109:131-40.
39. Jain G, Schwinn KE, Gould KS. Betalain induction by I-DOPA application confers photoprotection to saline-exposed leaves of Disphyma australe. New Phytol. 2015;207:1075-83.

40. Polturak G, Aharoni A. "la vie en rose": biosynthesis, sources, and applications of betalain pigments. Mol Plant. 2018;11:7-22.

41. Stracke R, Holtgrawe D, Schneider J, Pucker B, Sorensen TR, Weisshaar B. Genome-wide identification and characterisation of R2R3-MYB genes in sugar beet (Beta vulgaris). BMC Plant Biol. 2014;14:249.

42. Hatlestad GJ, Akhavan NA, Sunnadeniya RM, Elam L, Cargile S, Hembd A, et al. The beet $Y$ locus encodes an anthocyanin MYB-like protein that activates the betalain red pigment pathway. Nat Genet. 2014;47:92.

43. Cheng MN, Huang ZJ, Hua QZ, Shan W, Kuang JF, Lu WJ, et al. The WRKY transcription factor HpWRKY44 regulates CytP450-likel expression in red pitaya fruit (Hylocereus polyrhizus). Hortic. Res. 2017;4:17039.

44. Meyers BC, Axtell MJ, Bartel B, Bartel DP, Baulcombe D, Bowman JL, et al. Criteria for annotation of plant MicroRNAs. Plant Cell. 2008;20:3186-90.

45. Hua QZ, Chen CJ, Chen Z, Chen PK, Ma YW, Wu JY, et al. Transcriptomic analysis reveals key genes related to betalain biosynthesis in pulp coloration of Hylocereus polyrhizus. Front Plant Sci. 2015;6:1179.

46. Hua QZ, Zhou QJ, Gan SS, Wu JY, Chen CB, Li JQ, et al. Proteomic analysis of Hylocereus polyrhizus reveals metabolic pathway changes. Int J Mol Sci. 2016;17:1606.

47. Esquivel P, Stintzing FC, Carle R. Pigment pattern and expression of colour in fruits from different Hylocereus sp. genotypes. Innov Food Sci Emerg. 2007;8:451-7.

48. Woo KK, Wong FNF, Chua HSC, Tang PY. Stability of the spray-dried pigment of red dragon fruit [Hylocereus polyrhizus (weber) Britton and Rose] as a function of organic acid additives and storage conditions. Philipp Agric Sci. 2011:94:264-9.

49. Stintzing FC, Schieber A, Carle R. Identification of betalains from yellow beet (Beta vulgaris L.) and cactus pear [Opuntia ficus-indica (L.) mill.] by highperformance liquid chromatography-electrospray ionization mass spectrometry. J Agric Food Chem. 2002;50:2302-7.

50. Wybraniec S, Stalica P, Jerz G, Klose B, Gebers N, Winterhalter P, et al. Separation of polar betalain pigments from cacti fruits of Hylocereus polyrhizus by ion-pair high-speed countercurrent chromatography. J Chromatogr A. 2009;1216:6890-9.

51. Naderi N, Stintzing FC, Ghazali HM, Manap YA, Jazayeri SD. Betalain extraction from Hylocereus polyrhizus for natural food coloring purposes. J Prof Assoc Cactus. 2010;12:143-54.

52. Rebecca OPS, Boyce AN, Chandran S. Pigment identification and antioxidant properties of red dragon fruit (Hylocereus polyrhizus). Afr J Biotechnol. 2010; 9:1450-4.

53. Rebecca OPS, Harivaindaran KV, Boyce AN, Chandran S. Potential natural dye with antioxidant properties from red dragon fruit (Hylocereus polyrhizus). Acta Hort. 2010;875:477-85

54. Lim SD, Yusof YA, Chin NL, Talib RA, Endan J, Aziz MG. Effect of extraction parameters on the yield of betacyanins from pitaya fruit (Hylocereus polyrhizus) pulps. J Food Agric Environ. 2011;9:158-62.

55. Wu LC, Hsu HW, Chen YC, Chiu CC, Lin YI, Ho JAA. Antioxidant and antiproliferative activities of red pitaya. Food Chem. 2006;95:319-27.

56. Tenore GC, Novellino E, Basile A. Nutraceutical potential and antioxidant benefits of red pitaya (Hylocereus polyrhizus) extracts. J Funct Foods. 2012:4:129-36.

57. Garcia-Cruz L, Valle-Guadarrama S, Salinas-Moreno Y, Joaquin-Cruz E. Physical, chemical, and antioxidant activity characterization of pitaya (Stenocereus pruinosus) fruits. Plant Food Hum Nutr. 2013;68:403-10.

58. Yao FR, Zhu H, Yi C, Qu HX, Jiang YM. MicroRNAs and targets in senescent litchi fruit during ambient storage and post-cold storage shelf life. BMC Plant Biol. 2015;15:181

59. Li H, Mao WJ, Liu W, Dai HY, Liu YX, Ma Y, et al. Deep sequencing discovery of novel and conserved microRNAs in wild type and a white-flesh mutant strawberry. Planta. 2013;238:695-713.

60. Yang J, Liu X, Xu B, Zhao N, Yang X, Zhang M. Identification of miRNAs and their targets using high-throughput sequencing and degradome analysis in cytoplasmic male-sterile and its maintainer fertile lines of Brassica juncea. BMC Genomics. 2013;14:9.

61. Zhang XN, Li X, Liu JH. Identification of conserved and novel coldresponsive microRNAs in trifoliate orange [Poncirus trifoliata (L.) Raf.] using high-throughput sequencing. Plant Mol Biol Rep. 2014;32:328-41.

62. Stafford HA. Anthocyanins and betalains: evolution of the mutually exclusive pathways. Plant Sci. 1994;101:91-8. 
63. Ye YX, Hu GB, Li JQ, Qin YH, Gu WQ, Zhai X, et al. A new red-fleshed pitaya cultivar 'Guanhuahong'. Acta Hortic Sinica. 2018;45(11):2267-8.

64. Ye YX, Hu GB, Li JQ, Qin YH, Zhou YW, Zhai X, et al. A new white-fleshed pitaya cultivar 'Guanhuabai'. Acta Hortic Sinica. 2018;45(S2):2739-40.

65. Chen CB, Wu PY, Xie FF, Sun LY, Xing YM, Hua QZ, et al. Breeding of 'Hongguan No. 1' and 'Shuangse No. 1' Pitayas with superior quality. HortScience. 2018;53(3):404-9.

66. Han $X$, Yin H, Song X, Zhang Y, Liu M, Sang J, et al. Integration of small RNAs, degradome and transcriptome sequencing in hyperaccumulator Sedum alfredii uncovers a complex regulatory network and provides insights into cadmium phytoremediation. Plant Biotechnol J. 2016;14:1470-83.

67. Langmead B. Aligning short sequencing reads with Bowtie. Curr Prot Bioinform. 2010;11:11.7.

68. Chen CF, Ridzon DA, Broomer AJ, Zhou ZH, Lee DH, Nguyen JT, et al. Realtime quantification of microRNAs by stem-loop RT-PCR. Nucleic Acids Res. 2005;33:e179.

69. Liu YL, Wang L, Chen DJ, Wu XM, Huang D, Chen LL, et al. Genome-wide comparison of microRNAs and their targeted transcripts among leaf, flower and fruit of sweet orange. BMC Genomics. 2014;15:695.

70. Chen CB, Wu JY, Hua QZ, Tel-Zur N, Xie FF, Zhang ZK, et al. Identification of reliable reference genes for quantitative real-time PCR normalization in pitaya. Plant Methods. 2019;15:70.

71. Livak KJ, Schmittgen TD. Analysis of relative gene expression data using real-time quantitative PCR and the $2^{-\Delta \Delta C}$ method. Methods. 2001;25:402-8.

72. Sparkes IA, Runions J, Kearns A, Hawes C. Rapid, transient expression of fluorescent fusion proteins in tobacco plants and generation of stably transformed plants. Nat Protoc. 2006;1:2019-25.

\section{Publisher's Note}

Springer Nature remains neutral with regard to jurisdictional claims in published maps and institutional affiliations.

Ready to submit your research? Choose BMC and benefit from:

- fast, convenient online submission

- thorough peer review by experienced researchers in your field

- rapid publication on acceptance

- support for research data, including large and complex data types

- gold Open Access which fosters wider collaboration and increased citations

- maximum visibility for your research: over $100 \mathrm{M}$ website views per year

At $\mathrm{BMC}$, research is always in progress.

Learn more biomedcentral.com/submissions 